\title{
ABSTRACTION AND SPECIFICATION \\ OF LOCAL AREA NETWORKS
}

\author{
By \\ Chang-Hyun J্ত \\ Bachelor of Economics \\ Sung Kyun Kwan University \\ Seoul, Korea \\ 1984
}
Submitted to the Faculty of the Graduate College of the oklahoma state University in partial fulfillment of the requirements for the Degree of MASTER OF SCIENCE July, 1988




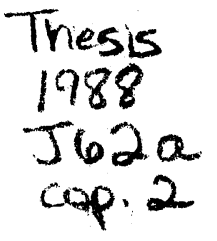




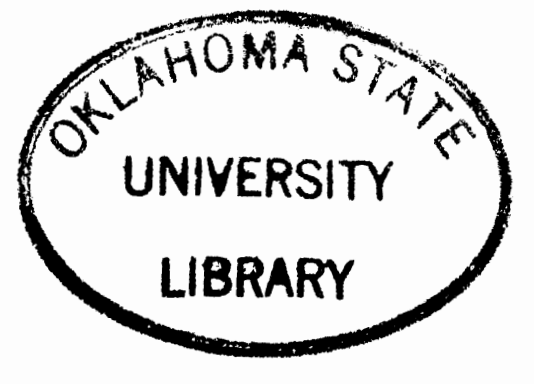

ABSTRACTION AND SPECIFICATION

OF LOCAL AREA NETWORKS

Thesis Approved:

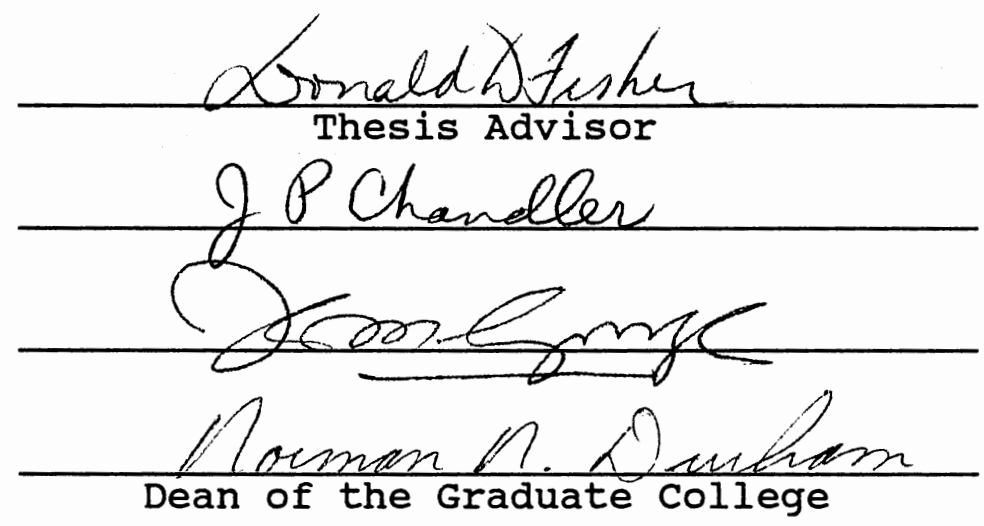

ii 


\section{ACKNOWLEDGMENTS}

I wish to express sincere appreciation to my advisor, Dr. D. D. Fisher for his warm encouragement and helpful advisement throughout my graduate study. I extend sincere thanks to committee member, Dr. K. M. George who provided valuable suggestions and comments on this thesis. I would also like to express my gratitude to $\mathrm{Dr}$. J. P. Chandler for his invaluable support and advisement. The helpful comments of Dr. C. Hutchens on LANs are also sincerely appreciated. I am also grateful to $\mathrm{Dr}$. M. Samadzadeh for his warm and constant guidance.

Throughout my life, my parents provided constant support and understanding without which this thesis could never have been done.

My deepest appreciation is extended to my wife, AeKyung and to my sons, Hyun-Soo and Jin-Soo for their patience and love. 
TABLE OF CONTENTS

Chapter

Page

I. INTRODUCTION . . . . . . . . . . . . . . . . 1

II. ABSTRACT SPECIFICATION . . . . . . . . . . 4

2.1 Abstraction . . . . . . . . . . . . . 4

2.2 Specification . . . . . . . . . . . 5

2.3 Abstract Data Types . . . . . . . . . 5

2.3.1 Data Types . . . . . . . . . . . 5

2.3.2 Abstract Data Types . . . . . . 6

2.4 The Characteristics of Abstraction . . . . 7

2.4.1 Data Abstraction . . . . . . . 8

2.4.2 Procedural Abstraction . . . . . 8

2.4.3 Behavioral Abstraction . . . . . 8

2.5 Abstract Specifications . . . . . . . 8

2.5.1 Algorithmic Specification . . . . 9

2.5.2 Operational Specification . . . . 10

2.5.3 Logical Specification . . . . . 10

2.5.4 Functional specification . . . . 10

2.5.5 Algebraic Specification . . . . . 10

2.6 Abstract Model .. . . . . . . . . . 11

2.7 Abstract Specification......... . 11

III. LOCAL AREA NETWORKS • . . . . . . . . . 13

3.1 Purpose of LAN • . . . . . . . . . . . . 13

3.2 LAN Standards . . . . . . . . . . . . 14

3.3 LAN Topologies . . . . . . . . . . . 15

3.3.1 Ring Topology . . . . . . . . . 17

3.3.2 Bus Topology . . . . . . . . . . . 17

3.3.3 Star Topology . . . . . . . . . 17

3.4 LAN Transmission Media . . . . . . . . 17

3.5 LAN Architecture . . . . . . . . . . 18

3.6 Internetwork Communication . . . . . 20

IV. ABSTRACTION OF LOCAL AREA NETWORKS . . . . . . 22

4.1 Abstract Model of LAN . . . . . . . . . . 24

4.2 The Formal Model of LAN Communication . . 27

4.3 The Formal Model of LAN Objects . . . . 31 
V. SPECIFICATION FOR IIAN ABSTRACTION . . . . . . 34

5.1 LAN Abstract Specification Template . . . 34

5.2 LAN Abstract Specification Examples . . . 36

VI. LAN ABSTRACT SPECIFICATION APPLICATION • • • . 48

6.1 Application to OSI Model IAN . . . . . . . 48

6.2 Application to DOD Model IAN . . . . . . 54

VII. CONCLUSIONS • • • • • • • • • • • • • 57

BIBLIOGRAPHY • • • • . • . • • • • • • . • . 59

APPENDIXES . . . . . . . . . . . . . . . . 63

APPENDIX A - OSI MODEL ABSTRACT MODULES • • • . 64

APPENDIX B - DOD MODEL ABSTRACT MODULES • • • 71 
LIST OF FIGURES

Figure

Page

1. Abstract Modeling . . . . . . . . . . . . 11

2. Data Communication Model . . . . . . . . 13

3. Seven-Layer OSI Reference Model . . . . . . . 14

4. Network Topologies . . . . . . . . . . . 16

5. LAN Architecture . . . . . . . . . . . . . 19

6. Homogeneous Inter-Network . . . . . . . . . 21

7. Heterogeneous Inter-Network . . . . . . . . 21

8. Inter-Network Data Packet Format . . . . . . . 21

9. Communication System . . . . . . . . . . 23

10. LAN Abstract Modeling . . . . . . . . . . . 24

11. Inter-Layer Communication Relation Graph . • . 25

12. Inter-Layer Communication Relation Matrix . . . . 26

13. Formal Model of LAN . . . . . . . . . . . . 32

14. LAN Abstract Specification Template . . . . . . 35

15. Relations among Object Modules . . . . . . . 36

16. Abstract Specification for OSU_LAN • . . • . • 39

17. Abstract Specification for LAN status . . . . . 40

18. Abstract Specification for object File . . . . 41

19. Abstract Specification for Object Message . . . 42

20. Abstract Specification for Object Batch_Job . • 43

21. Abstract Specification for object PDU . . . . . . 44

22. Abstract Specification for Object Buffer . • . 45 
23. Relations between Service and Abstraction . . . 47

24. Operations in Connection-Mode Service . . . . . 50

25. IAN Communication Service . . . . . . . . 51

26. Trace of Service . . . . . . . . . . . 53

27. DOD Communication Architecture for LAN . . . . 55

28. Trace of DOD Communication . . . . . . . . 56 
CHAPTER I

\section{INTRODUCTION}

An abstraction is a methodology for program construction. A large program problem can be decomposed into a number of small programs called modules. Abstraction is a way to do decomposition. Using an abstraction methodology, complicated objects can be simplified by decomposing the original objects into sub-modules until we can abstract each module as a specific function. We use here three kinds of abstractions [Liskov 1986]. Data types are the kinds of data such as integer, real, logical and character. Data objects are the elements in the set of data. Data structures consist of the operations applicable to the data objects and the data objects themselves. An abstract data type is a the mathematical model with a collection of operations defined on the data objects [Cleaveland 1986] [Guttag 1980]. Data abstraction allows us to defer decisions about a data structure during implementation until the data structure is fully specified. A procedural abstraction is a mapping from the arguments to the results with possible modification of some of the arguments. A behavioral or a functional abstraction is a description of the behaviors of modules when they are 
invoked. An abstraction is intangible. We have some difficulties to understand what an abstraction is, without any description. The specification is this description. A specification is the only tangible record of an abstraction.

A local area network(LAN) is a communications network that provides interconnection of a variety of data communicating devices within a small area [Stallings 1987]. LAN makes possible a form of computing that is distributed in several ways and provides several communications media and channels for data, images, and voice communication.

Specification for a computer network protocol is a formal description of its service function. Recently, a few papers introduced the specification, testing and verification techniques for distributed systems and communication protocols [Gehani 1986] [Lam 1984] [Sunshine 1981]. Those are formally described by Petri Net models, attributed grammars and some dedicated languages. However, those are not machine-readable, hence they cannot be normally used as input to design automation or simulation of network systems.

In this paper, we use the abstraction methodology to describe local area networks. This work includes an abstract model for a IAN and the specification of LAN communication protocols using a specification template developed based on the LAN abstract model. The advantage of such an approach is that the specification could be 
machine-readable. In LAN abstraction, we discuss the functional properties of a data structure and its operations, then specify it in abstract template constructs. This paper includes an abstract specification of the functional capabilities of its physical components, the data structure, the nature of control and information flow between components in local area networks. Chapter 2 deals with general concepts for abstraction and specification. Chapter 3 provides an overview of local area network. Chapter 4 introduces LAN abstract model and the formal model of LAN communication and objects. Chapter 5 presents the specification template and its examples. Further applications of abstract specification to OSI model LAN and DOD model LAN are shown in Chapter 6. This paper ends with the conclusions in Chapter 7. 


\section{CHAPTER II}

\section{ABSTRACT SPECIFICATION}

\subsection{Abstraction}

An abstraction provides a systematic tool used in classifying and solving problems. Abstraction is a mathematical modeling of the system. Discussion about abstraction is also becoming abstract. To avoid this abstraction, a formal or an informal model is used. The functions of the system are fixed and are already defined. The complexity of the functions, however, is beyond comprehension of the users who manipulate the system functions and even the implementors who facilitate the system. Using abstraction, complexity is reduced and the problem is generalized in the process of reducing the redundancy and omitting irrelevant details and reconstructing ambiguous flow. A good abstraction is well balanced between the level of the abstraction and its complexity. It must also have a good balance between the machine dependent modeling and the machine independent modeling. Some abstractions are portable if they can be implemented on several independent systems without losing compatibility. The harmonious abstraction between the 
logical environment and the physical environment is a very difficult problem.

\subsection{Specification}

Specification for a computer network protocol is a formal description of its service function. A number of communication protocol specification techniques are as follows [Gehani 1986] [Guttag 1978] [Sunshine 1981]:

1) abstract machine model,

2) formal languages model,

3) Petri Nets model,

4) abstract data types.

Not only can abstract machine automata diagram easily network structures [Bochmann 1978], but Petri Nets also describe well abstract concurrent systems [Merlin 1979] [Keller 1976]. However, those are not machine-readable, hence they cannot be normally used as input to design automation or simulation of network systems. Here we show how to use abstraction methodologies for the specification of communication systems in terms of local area networks which can be a machine-readable input for network software design with some proper modification.

\subsection{Abstract Data Types}

\subsubsection{Data Types}

Data types are specific programming language 
constructs used to describe and define data structures. A type is a set of values. A data type is a set of values and a set of operations on the values. Types specify how to interpret the values. The relationships between the different types depend on the point of view. Every object belongs to exactly one type, however, objects of one type can represent objects of other types. Types are helpful to understand objects and to detect errors. (Type checking is one of the most powerful error detection capabilities of a compiler.)

\subsubsection{Abstract Data Types}

Abstract data types(ADT) were probably the most important advance in programming languages during the 1970's [Cleaveland 1986] [Guttag 1980]. The major conceptual idea of ADT is to separate the use of a type from the representation and implementation of a type. The use of a type should depend on the set of values and operations. It should not depend on either its representation or its implementation. The use of data types can be specified by the syntax and semantics. The languages provide a rich syntax for expressing data types and ADT. An abstract data type may have various implementations. Interchangeability of implementations is another merit of abstract data types. Since it is not necessary that the application programmer need to know the encapsulated algorithms, it is only necessary for him to learn how to 
specify the interface parameters which is generally a much simpler task. This encapsulation is a major merit of abstract data types.

\subsection{The Characteristics of Abstraction}

Abstraction provides a way of organizing and designing programs that are both more reliable and easier to change. We cannot call just a collection of related procedures a data abstraction. A merit for using abstraction is the inter-changeability of implementation. If a more efficient implementation is found it can readily be substituted for the older implementation. Three kinds of abstractions are used for an abstraction here. Those are data abstraction, procedural abstraction and behavioral abstraction. Liskov [1986] describes well about data abstraction and procedural abstraction. But how does behavioral abstraction differ from those two kinds of abstraction? Behavioral abstraction is a high level language like description of an invoked module which consists of data abstraction and procedural abstraction. When such a module is invoked, necessary operations are held spontaneously. If the abstract module is invoked as a main module, such an abstraction is absolutely necessary. An abstraction consists of two parts. The first part is the specification and the second one is the implementation. In this paper, we introduce the specification scheme with an abstraction model of a IAN, and then we show how this specification technique is used 
for the implementation.

\subsubsection{Data Abstraction}

Data abstraction consists of a set of objects and a set of operations characterizing the behavior of the objects. Data abstraction separates the use of a data type from the implementation of a data type.

\subsubsection{Procedural Abstraction}

A procedure provides a transformation from input arguments to output arguments. Procedural abstraction allows us to explain data objects in terms of input and output parameters.

\subsubsection{Behavioral Abstraction}

Behavioral abstraction specifies the behaviors of invoked modules in which both data abstraction and procedural abstraction are composed. When each module is invoked by calling its quantifiers, its functional behavior is defined by its behavioral abstraction.

\subsection{Abstract Specifications}

A specification says exactly what a data type is and how its operation behaves. This information enables a programmer to implement the data type and the operation, and it enables users to use the data type and the operations. Precision and communication are the two most 
important qualities of specifications to understand and to interpret; specifications must be precise and unambiguous. Specifications are used to communicate between the user and the implementor. Natural language is easier to read but not precise, and most formal languages are precise but difficult to read. Precision and readability are conflicting goals of abstract specifications. Abstract specification provides a means for defining abstractions. There are are several methods for defining abstractions including the following [Cleaveland 1986].

1) Algorithmic specification

2) Operational specification

3) Logical specification

4) Functional specification

5) Algebraic specification

Now let us illustrate how to define the abstractions in terms of each specification.

\subsubsection{Algorithmic Specification}

The algorithmic specification can define the data types and the operations as a plain English sentence which is a brief and precise description.

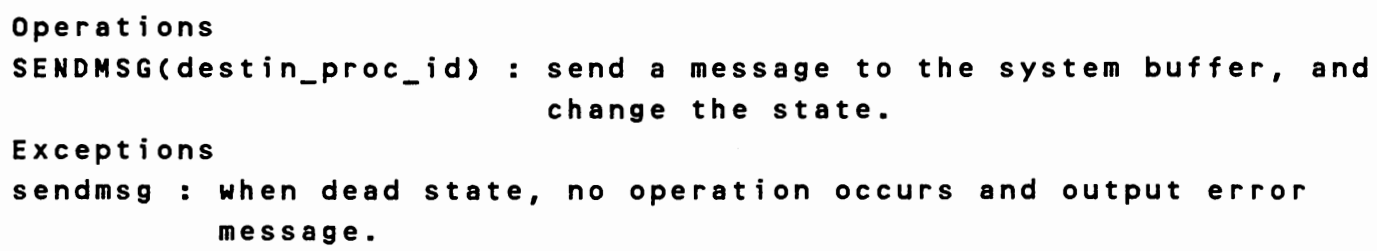




\section{5 .2 Operational Specification}

The operational specification is the most high_level language like implementation.

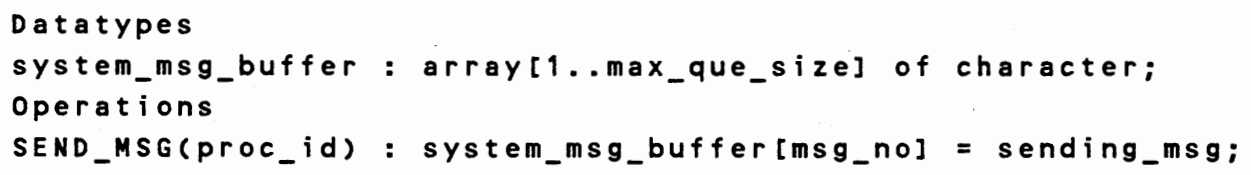

\subsubsection{Logical Specification}

Logical specifications use input and output assertions written in predicative calculus to describe the conditions before and after execution of statements and procedures.

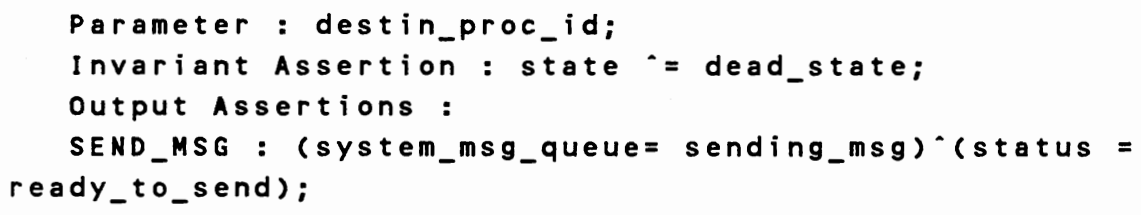

\subsubsection{Functional Specification}

Functional specifications describe the output in terms of input using mathematical constructions such as sets, functions, and sequences for representing objects traditionally.

\section{system_msg_buffer = set of messages;}

SENDMSG : message $x$ system_msg_buffer $\rightarrow$ system_msg_buffer;

\subsubsection{Algebraic Specification}

The algebraic approach views data types as algebra, and to specify a type the axioms that describe such an algebra can be used. 


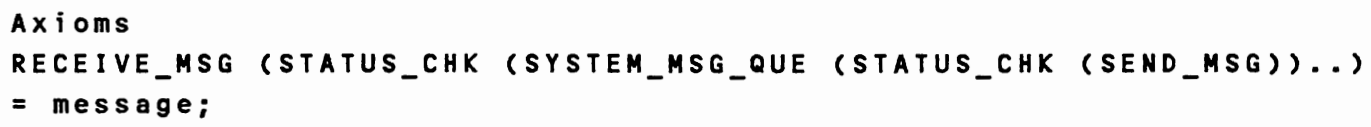

\subsection{Abstract Model}

In the context of a formal model, the abstraction for a certain problem solving can be done as illustrated in Figure 1.

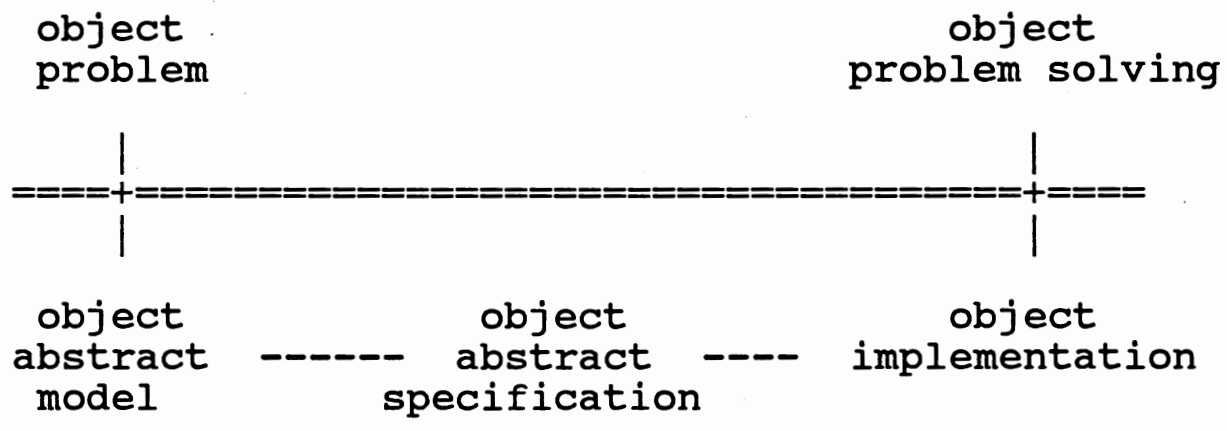

Figure 1. Abstract Modeling

Properly combining all of the above methods to specify abstractions, we give an abstract model of LAN for its communication and its objects in Chapter 4 after we review some IAN models in Chapter 3.

\subsection{Abstract Specification}

The abstract specification describes how all the operations work. It can be a procedural abstraction and it 
may be represented by a programming language. An abstract specification may be implemented in various ways. The inter-changeability of certain implementations and the encapsulation of data objects are the major motivations of the abstraction. The abstract specification of LAN systems are shown in Chapter 5 and 6. 
CHAPTER III

LOCAL AREA NETWORKS

\subsection{Purpose of IAN}

A local area network is a communications network that provides interconnection of a variety of data communicating devices within a small area [Stallings 1987]. The main purpose of a IAN is for distributed computing and resource sharing among associated devices. LAN makes possible a form of computing that is distributed in several ways and provides several communications media and channels for data, images, and voice communication [Figure 2].

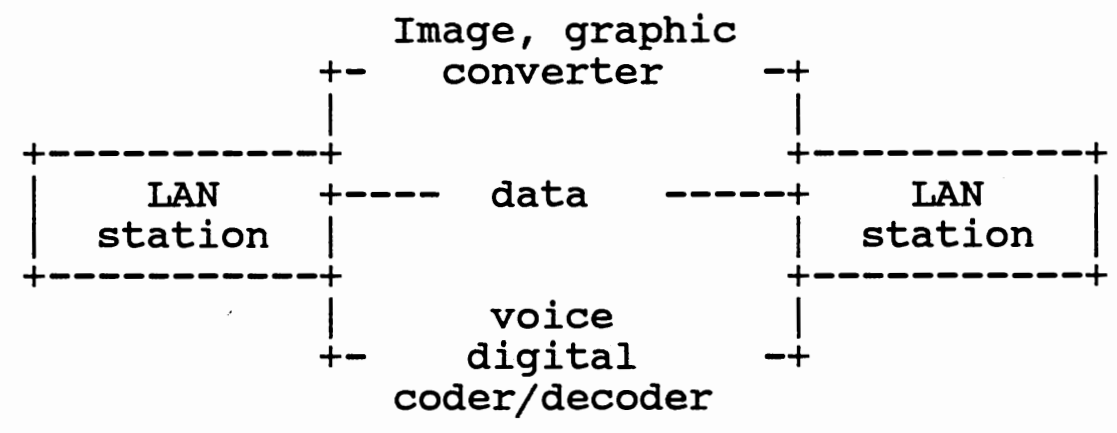

Figure 2. Data Communication Model 


\subsection{LAN Standards}

In general LAN computers from different vendors are different from each other, thus it is desirable to have a set of standards for IAN. All specifications for LAN in this paper try to follow the osI(Open systems Interconnection) Reference Model standardized by International Organization for standardization(ISO) and IEEE 802 LAN standards. The OSI Reference Model [ISO 1981] contains the following seven layers [Figure 3].

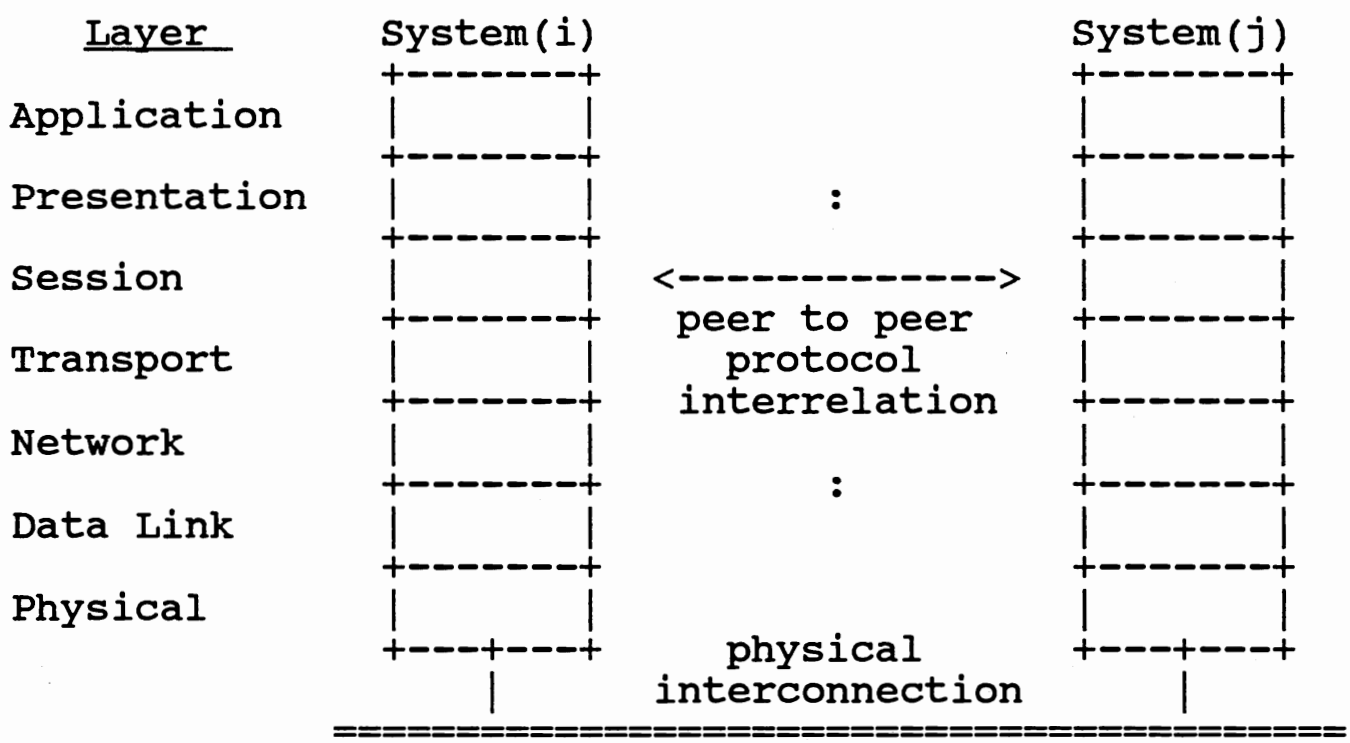

Figure 3. Seven-Layer OSI Reference Model

Protocols are the rules that communicating processes follow when they exchange messages and control information. 
The protocols specify the format of the data and control information and give the procedures that the sender and receiver follow. Protocols are characterized by a cost and a reliability.

\subsection{LAN TOpologies}

Topology refers to the way in which the end points, or stations, attached to the network are interconnected. The common LAN topologies are the following [Figure 4].

1) Ring network

2) Bus network

3) Star network

4) Tree network

5) Mesh network

Among those topologies, the standardizations of I.AN focus on the bus, ring and star topologies. Bus networks use the Carrier Sense Multiple Access with Collision Detection (CSMA/CD) scheme, while the ring network uses token passing scheme. A transmission scheme says how to control the transmission of traffic in a network. Two transmission techniques are used for the bus topology: baseband and broadband. Baseband uses digital signaling on twisted pair wire or coaxial cable. Baseband transmission is bidirectional. A broadband scheme allows many different stations to have messages in the network at the same time. Broadband uses analog signaling on coaxial cable. It covers much greater distance than baseband. But unlike baseband, 
broadband is unidirectional transmission scheme. The ring topology is major alternative of the bus topology. The ring consists of a number of repeaters connected on unidirectional transmission links. Data are transmitted in packets and transferred sequentially around a ring.

Ring

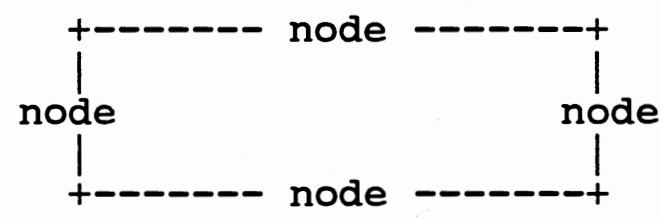

Bus

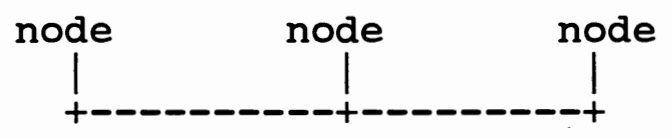

Star

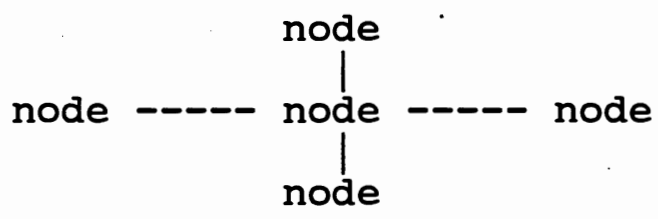

Tree

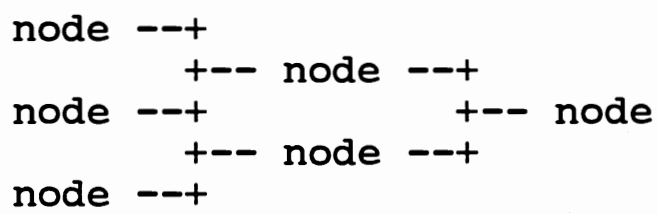

Mesh

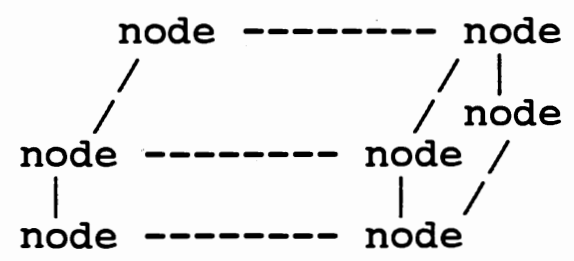

Figure 4. Network Topologies 


\subsubsection{The Ring Topology}

A ring network consists of nodes that are connected by transmission links to form a series of nodes in a $\operatorname{link}($ loop)

\subsubsection{The Bus Topology}

The nodes are attached to a bus that provides a bidirectional transmission facility.

\subsubsection{The star Topology}

All nodes are connected to a centralized controller in which nodes exchange data through the central node.

\subsection{LAN Transmission Media}

The transmission medium is the physical path between transmitter and receiver. The media used for local area networks are twisted pair, coaxial cable and optical fiber. A twisted pair is used for normal voice communication. Twisted pair can be a low-cost solution for a small network, but the transmission distance for signals on twisted pair is relatively short without the aid of repeaters. A coaxial cable can be used for transmitting both analog video and digital data signals. Coaxial cable has a much wider bandwidth. The shield on a coaxial cable is used to reduce noise intrusion. Since it is easy to install and it supplies a higher transmission speed, it is 
widely used in cable TV industry. Optical fiber media is now used in IAN systems with several advantages over other media. It has lower power loss, and greater bandwidth potential. But optical fiber is more expensive than other transmission medium.

\subsection{LAN Architecture}

Computer networks consist of the host and the subnets. The subnets include the switching elements and the transmission line. Most computer networks are organized in layers or levels, each built upon its predecessor level. A computer network consists of two layers, a physical

communication level and a virtual communication level. The virtual communication level is divided into several layers like the OSI model. Each layer performs a certain task and provides services for the next layer. The set of interaction rules between the layers is called a protocol. The functions performed by each layer are the following.

1) Initiation of entity interaction

2) Data transmission

3) Data manipulation

4) Information control

5) Interaction termination

Figure 5 shows a typical IAN architecture. 
Typical LAN:

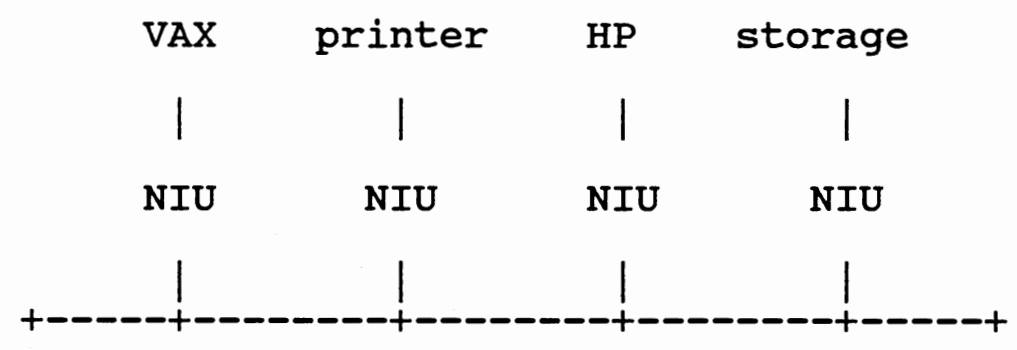

physical medium

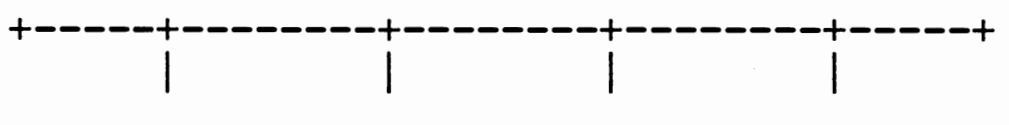

Personal Computer LAN :

$\begin{array}{cccc}\text { NIU } & \text { NIU } & \text { NIU } & \text { NIU } \\ \text { PC } & \text { I } & \mid & \mid \\ \text { I } & \text { I } & \text { PC } & \text { PC } \\ \text { hisk } & \begin{array}{c}\text { floppy } \\ \text { disk }\end{array} & \text { printer } & \text { modem } \\ & & & \mid \\ & & & \text { remote } \\ \text { main frame }\end{array}$

NIU: Network Interface Unit

Figure 5. LAN Architecture 


\subsection{Internetwork Communication}

Sometimes it is necessary that a IAN access another network like a nation-wide network as well as another IAN. A network interface unit(NIU) implements and provides an interface capability for interconnection between nodes in different IANs. It acts as a communication controller. A network interface unit can support several communication terminals. An intermediate node which provides an interface between a IAN and another IAN or a WAN(Wide Area Network) for communication is called a bridge or a gateway. A bridge consists of two network interface units linked together. A bridge serves as a connector to connect separate homogeneous networks [Figure 6]. A gateway is also a device for connecting two systems that use different protocols [Figure 7]. It behaves as a protocol converter. Thus a bridge may not change the format of protocol content while a gateway may modify the protocol format. An example of an inter-network data packet is given in Figure 8. The internetwork protocol has responsibility for multiplexing and demultiplexing a data packet. The well known inter-network protocol is Internet Protocol(IP) which was developed by ISO and by DOD and it is used with Transmission Control Protocol(TCP). IP provides a connectionless data transfer service to other IP users. 


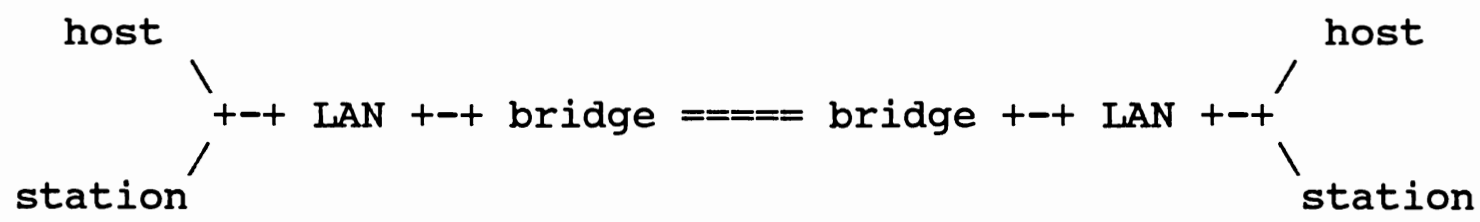

Figure 6. Homogeneous Inter-network

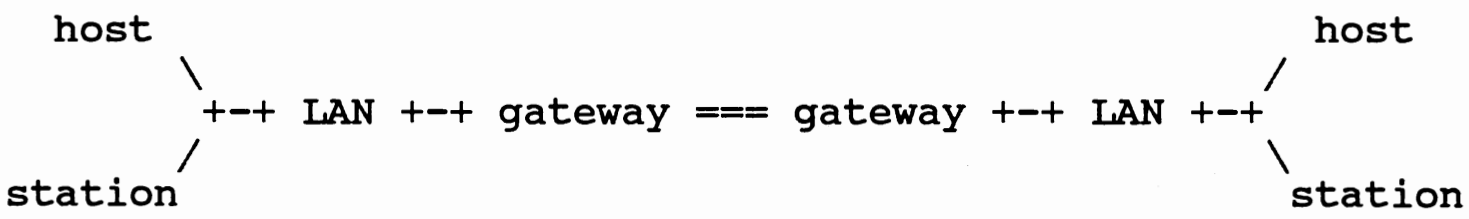

Figure 7. Heterogeneous Inter-network

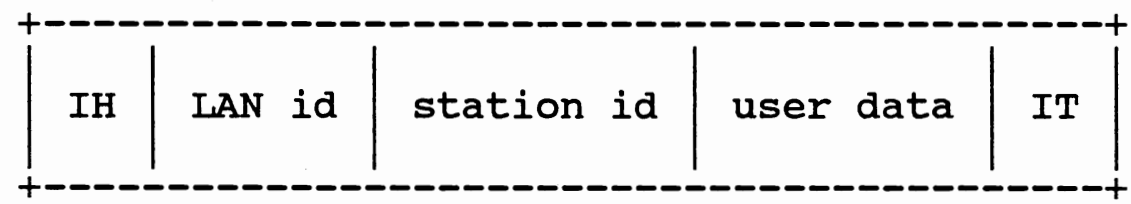

IH : inter-network data packet header

IT : inter-network data packet trailer

Figure 8. Inter-Network Data Packet Format 
CHAPTER IV

\section{ABSTRACTION OF LOCAL AREA NETWORKS}

Using protocols, two users can communicate with each other from different IAN stations [Figure 9]. Protocols involve interactions with users or programs in order to get certain functions performed. How these functions are actually performed by the protocol is not really of concern; only the end result matters. The users regard the protocols as black boxes. Each protocol level makes use of the services provided by the next lower level. Data transmission is accomplished by passing data between adjacent lsyers. We call this an inter-layer transmission. In this chapter, we discuss an abstract modeling of LAN communication using such an inter-layer data transmission. A formal model of IAN data objects are also described. 


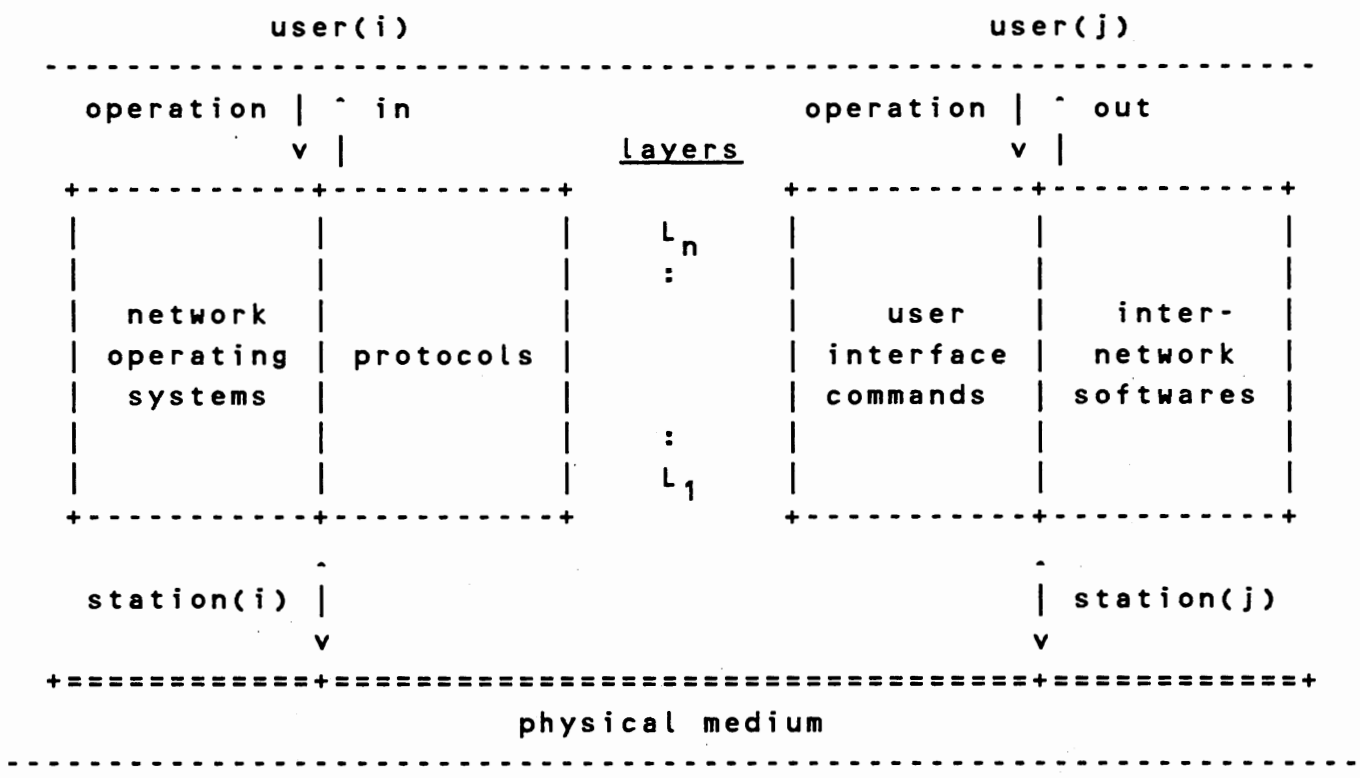

Figure 9. Communication System 


\subsection{Abstract Model of IAN}

LAN abstract model consists of an abstraction of LAN communication and a formal model for LAN objects. A LAN abstraction can be modelled as illustrated in Figure 10. In this section we describe basic concepts for LAN abstact modeling. Then the formal model of abstract LAN communication in terms of inter-layer transmission and abstract modeling for data object used in a LAN are followed in the next sections.

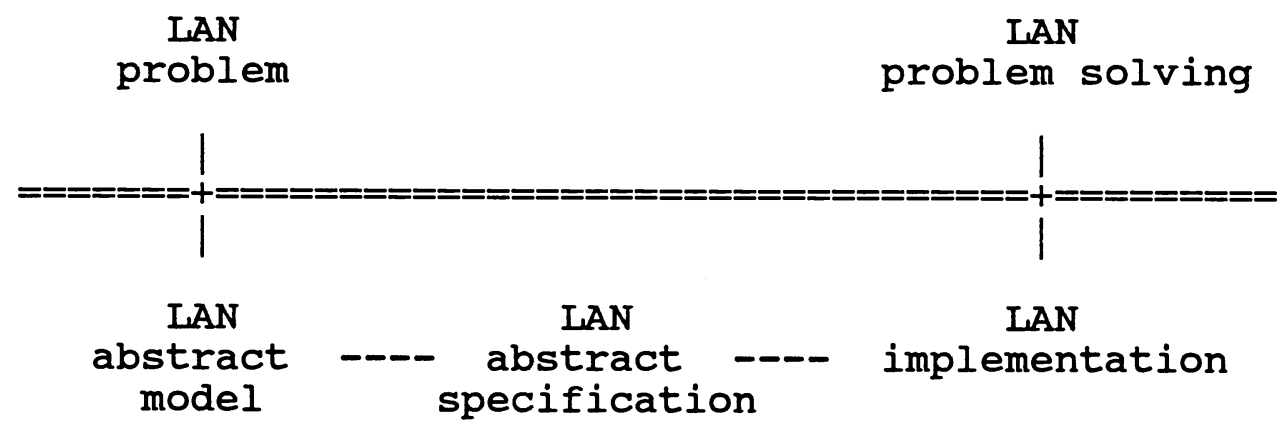

Figure 10. IAN Abstract Modeling 
In the layer set of $\mathrm{L}$, there are upward and downward inter-layer communication relations. Suppose $\mathrm{L}$ is an ordered set of layers, and let $\mathrm{R}$ represent an inter-layer communication relation on the set $L$. Thus $R$ is

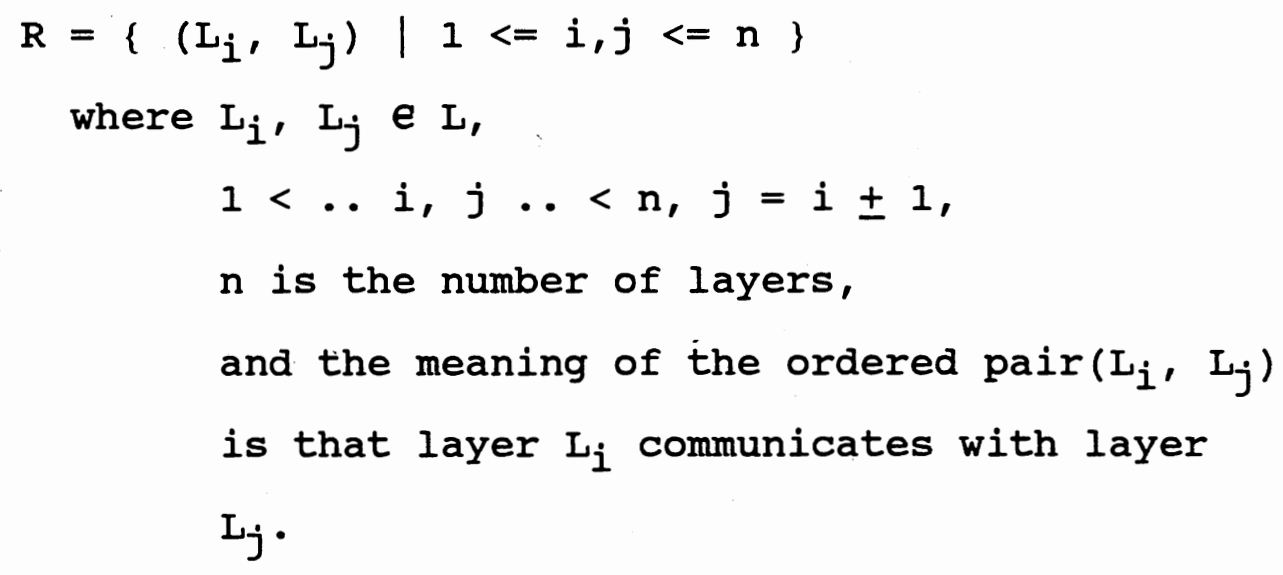

The directed graph representation of the bidirectional inter-layer communication relations $R\left(L_{i}, L_{j}\right)$ is the following [Figure 11].

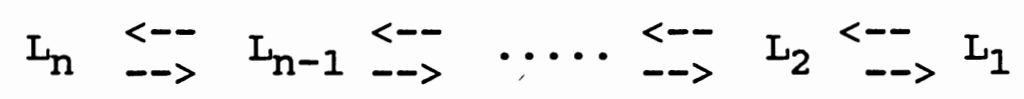

Figure 11. Inter-Layer Communication Relation Graph 
For a particular local area network, the matrix representation $\mathrm{M}$ of these relations is

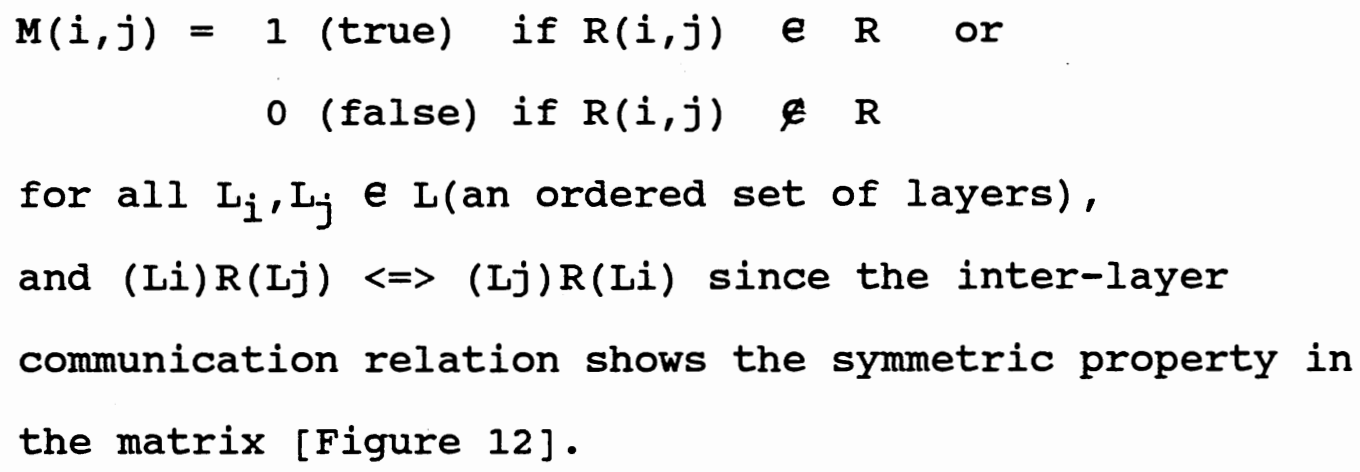

L1 L2 L3 L4 L5 L6 L7 $\ldots$

$\begin{array}{clllllll}\text { L1 } & 0 & 1 & 0 & 0 & 0 & 0 & 0 \\ \text { L2 } & 1 & 0 & 1 & 0 & 0 & 0 & 0 \\ \text { L3 } & 0 & 1 & 0 & 1 & 0 & 0 & 0 \\ \text { L4 } & 0 & 0 & 1 & 0 & 1 & 0 & 0 \\ \text { L5 } & 0 & 0 & 0 & 1 & 0 & 1 & 0 \\ \text { L6 } & 0 & 0 & 0 & 0 & 1 & 0 & 1 \\ \text { L7 } & 0 & 0 & 0 & 0 & 0 & 1 & 0 \\ : & & & & & & & \\ : & & & & & & & \end{array}$

Figure 12. Inter-Layer Communication Relation Matrix 
In LAN, the services are provided by interfaces between adjacent layers. To transfer data or control information, it is transferred to the next layer by invocation of the services of the next layer with given primitives. Transmitted data reaches the physical layer, and is delivered to a destination entity through the transmission medium. Upward service invocations are then invoked until the data finally arrives at the user interface layer in the destination entity. Now let us define the formal model of LAN communication in terms of such inter-layer service communication.

\subsection{The Formal Model of LAN Communication}

The formal model of LAN communication is defined in terms of inter-layer service communication described above. We need the following definitions:

1) L represents an ordered set of layers $\left\{L_{n}, L_{n-1}, \ldots, L_{j}, L_{i}, \ldots, L_{1}\right\}$ where $L_{n}$ is the highest layer and $I_{1}$ is the lowest layer;

2) II represents inter-layer communication-flow concatenation

( left-associative and non-commutative binary operator );

3) \$\$ represents inter-node physical connection;

4) \#\# represents inter-network communication connection; 
5) $\%$ represents inter-network converter

\{ gateway, bridge \};

6) $R=\left\{\left(L_{i}, L_{j}\right) \mid 1<=i, j \Leftrightarrow=n\right.$ and $\left.i=j \pm 1\right\}$ inter-layer communication relationship between layers $\mathrm{L}_{i}, \mathrm{~L}_{\mathrm{j}}$.

The meaning of the ordered pair $\left(L_{i}, L_{j}\right)$ is that layer $\mathrm{L}_{i}$ communicates with layer $\mathrm{L}_{j}$;

7) $\mathrm{D}=$ ( downward transmission, upward transmission \};

8) An ordered pair $(r, d)$ where $r \in R$ and $d \in D$ is called a transmission.

\section{Definition 2.1}

Communication in a homogeneous LAN can be represented by the string:

$$
\begin{aligned}
& \left(\left(L_{n}, L_{n-1}\right), R\right)||\left(\left(I_{n-1}, L_{n-2}\right), R\right)|| \ldots \mid \\
& \left(\left(L_{j}, L_{i}\right), R\right)|| \ldots||\left(\left(L_{3}, L_{2}\right), R\right)|| \\
& \left(\left(L_{2}, L_{1}\right), R\right) \quad \$ \$\left(\left(L_{1}, L_{2}\right), R\right) \|\left(\left(L_{2}, L_{3}\right), R\right) \\
& |\ldots .|\left(\left(L_{n-1}, L_{n}\right), R\right)
\end{aligned}
$$

Communication in a heterogeneous IAN can be represented by the string:

$$
\begin{aligned}
& \left(\left(I_{n}, L_{n-1}\right), R\right)||\left(\left(L_{n-1}, L_{n-2}\right), R\right)|| \ldots \ldots|| \\
& \left(\left(L_{j}, L_{i}\right), R\right)\|\ldots \mid\|\left(\left(L_{3}, L_{2}\right), R\right) \| \\
& \left(\left(L_{2}, L_{1}\right), R\right) \text { \#\# } \frac{8}{8} \#\left(\left(L_{1}, L_{2}\right), R\right) \| \\
& \left(\left(I_{2}, L_{3}\right), R\right) \| \ldots||\left(\left(I_{m-1}, I_{m}\right), R\right)
\end{aligned}
$$$$
\text { where (i) } n, m>0, n \text { and } m \text { need not be same and }
$$ 


\section{(ii) $\%$ (inter-network converter) may have some protocol layers.}

Definition 2.2

Let $T: R \times T \rightarrow\{T s, T e, T r\}$

$T=T s$ if transmission is successful, Te if an error occurs, $\operatorname{Tr}$ if re-transmission is needed. (Tr eventually is either Ts of Te.)

with the property, T distributes over '/|' in a communication.

With the above definition of $T$ we have the following:

$$
\begin{aligned}
& \text { if } c=s 1 \| s 2|| \ldots . \\
& \text { where } s i=\left(\left(L_{i}, L_{i \pm 1}\right), d\right) \\
& \text { then } T(c)=T(S I)|| T(s 2) \| \ldots . .
\end{aligned}
$$

\section{Definition 2.3}

A communication consists of a series of transmissions, such a communication can be defined by the concatenation of inter-layer transmissions:

The operation ' $\mid$ ' can be defined for values of $T$ as follows:

$$
\begin{aligned}
& \text { Ts } \| \mathrm{Ts}=\mathrm{Ts}, \\
& \mathrm{Ts} \| \mathrm{Tr}=\mathrm{Tr}, \\
& \mathrm{Ts} \| \mathrm{Te}=\mathrm{Te}, \\
& \text { undefined otherwise } \\
& \text { where left-associative and non-commutative }
\end{aligned}
$$


binary operator ' $\mid$ ' means inter-layer communication-flow concatenation.

We deduce the result of whole communication from substitution of partial inter-layer transmissions. If we have a communication of

$$
\text { Ts }\|\operatorname{Tr}\| \mathrm{T}(\mathrm{Si}) \| \mathrm{T}(\mathrm{Sj}) \ldots .
$$

By substitution, it would be

$$
\operatorname{Tr}\|\mathrm{T}(\mathrm{Si})\| \mathrm{T}(\mathrm{Sj}) \ldots . .
$$

If $\operatorname{Tr}$ is eventually $\mathrm{Te}$, then the string is

$$
\text { Te }\|\mathrm{T}(\mathrm{Si})\| \mathrm{T}(\mathrm{Sj}) \ldots . .
$$

Here, we do not have any definition for ( Te $\mid$...) , it means such a transmission is out of the question; Note that the transmission to the next adjacent layers is impossible after the transmission error between the certain layers. Non-definition stands for an impossible transmission or a transmission error. The result of the above transmission is

$\mathrm{Te} \quad \ldots$.

which indicates a transmission error.

What is the valid communication in terms of the data transmission T? A communication is successful if and only if a transmission stream reduced to Ts. If we have the transmissions

$$
\text { Ts }\|\operatorname{Tr}\| \mathrm{T}(\mathrm{Si}) \| \mathrm{T}(\mathrm{Sj}) \ldots
$$

in a certain communication, such transmissions can be reduced by substitution of transmission definitions.

$$
\operatorname{Tr} \quad\|\mathrm{T}(\mathrm{Si})\| \mathrm{T}(\mathrm{Sj}) \ldots
$$




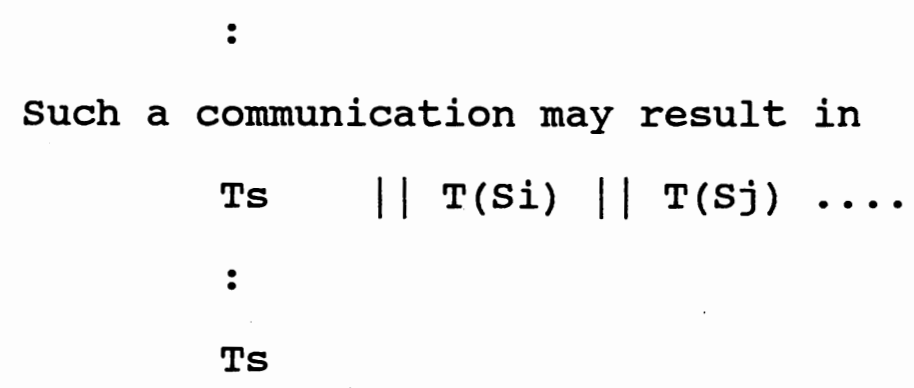

which means a valid communication with the re-transmission during the data transmissions.

So far, we have defined an abstract LAN model using the mathematical modeling methodologies. In the next section we discuss the formal model of LAN objects.

\subsection{The Formal Model of LAN Objects}

Now we introduce the formal model of LAN objects [Figure 13]. Data packet, file, message buffer and status vector are the LAN objects. The model is based on the elements of a LAN such as communication layers, topology, transmission medium, data structure of LAN objects and communication details.

In this LAN formal model, the topologies and media used in a LAN can be defined in 'TOPOLOGY' and 'MEDIUM'. 'DATA STRUCTURE' defines the data and data type used in a LAN structure. 'COMMUNICATION LAYERS' shows a LAN structure which consists of several layers. Transmission scheme, the functions of layers, and the primitives used with the layers can be described in 'COMMUNICATION DETAIL'. In Figure 13, '->' and ' $<-1$ mean downward and upward transmissions respectively. 


\section{ABSTRACT_LAN}

$$
\begin{aligned}
& \text { TOPOLOGY } \quad \Rightarrow \text { topology list } \\
& \text { topology_list } \Rightarrow\{\text { ring, bus, star \} }
\end{aligned}
$$

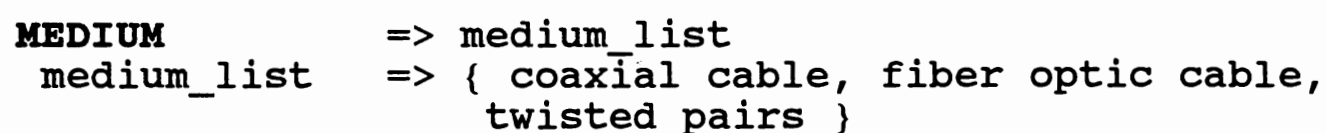

$$
\begin{aligned}
& \text { DATA STRUCTURE }=>\text { data_list } \\
& \text { data_list } \Rightarrow \text { data }^{-} \text {: data_type } \\
& \text { COMMUNICATION IAAYERS } \Rightarrow \text { layer_list } \\
& \text { layer_list } \Rightarrow\left\{I_{n}, I_{n-1}, \ldots, I_{j}, I_{i}, \ldots, I_{1}\right\} \\
& \mathrm{L}_{\mathrm{n}}: \text { highest layer } \\
& L_{i}, L_{j}: \text { middle layers } \\
& \mathrm{L}_{1} \text { : physical layer } \\
& \text { COMMUNICATION DETAII } \Rightarrow \text { communication_flow } \\
& \text { communication_flow } \Rightarrow\left\{\left(I_{n}, I_{n-1},\{->,<-\}\right)\right. \text {, } \\
& (\ldots, \ldots, \quad\{->,<-\}) \text {, } \\
& \left(L_{j}, L_{i}, \quad\{->,<-\}\right) \text {, } \\
& (\ldots, \ldots, \quad\{\rightarrow,<-\}) \text {, } \\
& \left.\left(I_{2}, I_{1}, \quad\{->,<-\}\right)\right\}
\end{aligned}
$$

END ABSTRACT_IIAN

$$
\text { Figure 13. Formal Model of IAN }
$$

Using this model, we introduce the IAN abstract specification template in which the LAN objects and their functions can be described [Figure 14 in Chapter 5]. The relationship between the IAN formal model and the LAN abstract specification template is that the IAN specification template is an instance of the LAN formal model. IAN specification template is high level language like. Each object or protocol can be specified using this template. Each specified template can be implemented in a module. Topology and medium in a formal model for a LAN can 
be specified in this template. To specify communication detail, necessary interface operations are modulized in a template. 


\section{CHAPTER V}

\section{SPECIFICATION FOR IAAN ABSTRACTION}

Now we combine properly abstract specifications which have been shown in Chapter 2, IAN in Chapter 3, and IAN abstraction and formal model of IAN in Chapter 4.

\subsection{LAN Abstract Specification Template}

Using the LAN formal model, we introduce the highlevel language like LAN abstraction template for specifying LAN objects. Figure 14 shows a template for an abstract specification which is associated with a LAN. There are seven dimensions in the space of an abstract specification for a LAN. A dimension of the network abstraction space relates to the distinction between structures and behaviors. The header 'ABSTRACT_IAN' introduces an abstract object name and 'OVERVIEw' defines an overall description and describes the operations with the objects. 'TOPOLOGY' shows all the possible topologies in the network structure and 'MEDIUM' specifies possible transmission media. 'DATA STRUCTURE' shows all the data objects and their types used in the module. 'INTERFACE' lists all the operations used for the interface in the object. 'OPERATION' specifies each operation involved in the abstract object. 'BEHAVIOR' 
describes the inherited functional behaviors of the modules when they are invoked. It is similar to the high-level language descriptions. A template ends with 'END ABSTRACT_IAN' trailer. Examples are shown in the next sections.

ABSTRACT_IAN object_name

OVERVIEW

abstract description of this module

TOPOLOGY

possible topologies (ring | bus | tree | star)

MEDIUM

possible media

(twisted pair | coaxial cable | optical fiber)

DATA STRUCTURE

data objects : data types

INTERFACE

interface operations

OPERATION

operation : procedural description

BEHAVIOR

functional abstraction of the object behavior

END ABSTRACT_ILAN

Figure 14. LAN Abstract Specification Template 


\subsection{LAN Abstract Specification Examples}

Any object in a LAN can be specified in the IAAN abstract specification template. The following example of an abstract specification for a subset of object modules to implement a LAN includes abstract modules named osu_lan, status, file, message, batch_job, pdu and buffer. Figure 15 shows the relations among these object modules by constructing a graph.

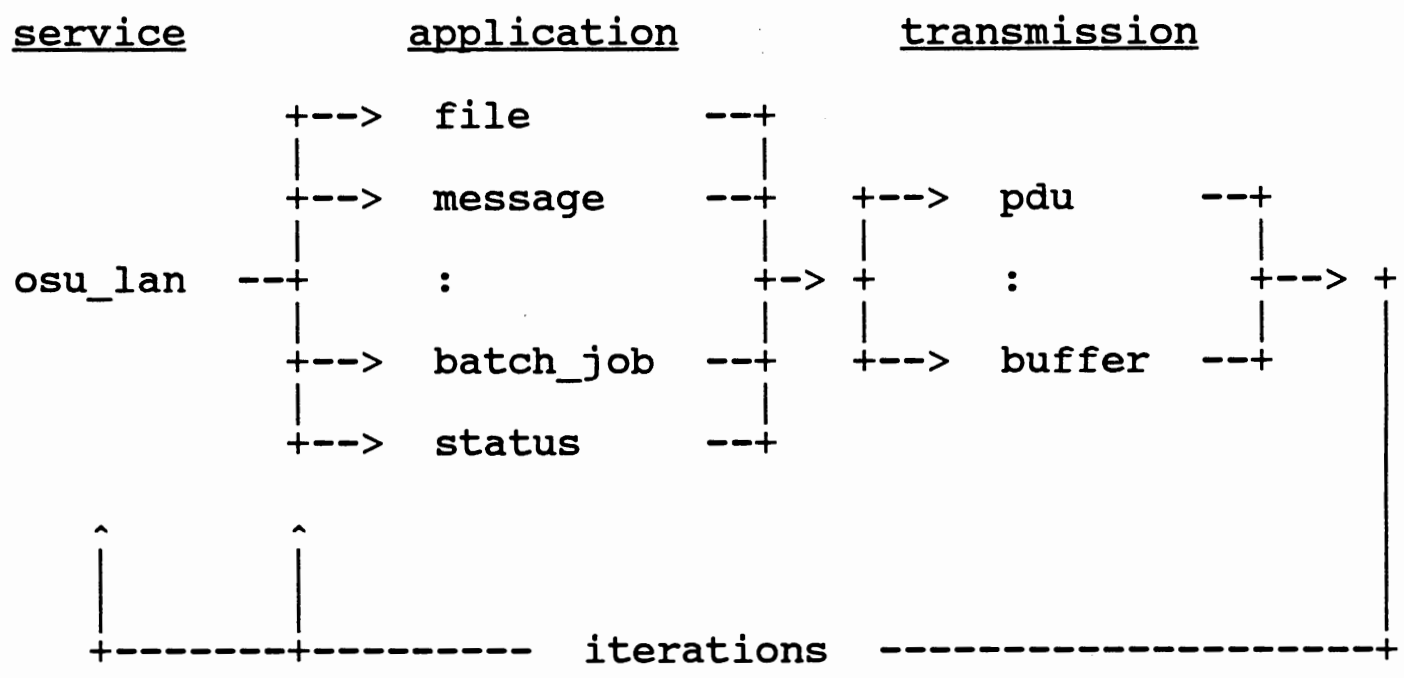

Figure 15. Relations among object Modules

In Figure 15, 'A $\rightarrow$ B' means that the module $A$ may call module B to request services or applications. 
In this example 'osu_lan' LAN abstract module can be interpreted to be a main module for implementation [Figure 16]

Actually, the application of abstract specification template to the logical or physical model leads to some problems. Not all the spaces can be specified well all the time. Then we left such a space as not applicable.

Also sometimes, we need access to LAN system status information. Then the module 'status' can be used [Figure 17]

The application layer of IAN supports file transfer [Figure 18], message transmission [Figure 19], and distributed batch jobs [Figure 20].

As we mentioned, there are seven layers in the OSI Reference Model. There is no direct communication between the peer layers except for the physical layer. Communication between applications need services of the lower layers. In the most common way, the data transmission between two entities is accomplished by both encapsulation and segmentation. A header is appended to or detached from the data as it is passed by each layer. The application layer of the receiving entity regains the same data type that the application layer of sending entity sent. This formatted data is called PDU(protocol data unit). PDU can be specified as shown in Figure 21.

In each layer and especially in the physical layer, the data to be transmitted may be delayed due to 
synchronization. A buffer is used for that purpose. Any waiting data can be buffered if necessary. The abstract module 'buffer' is called at that time [Figure 22]. 


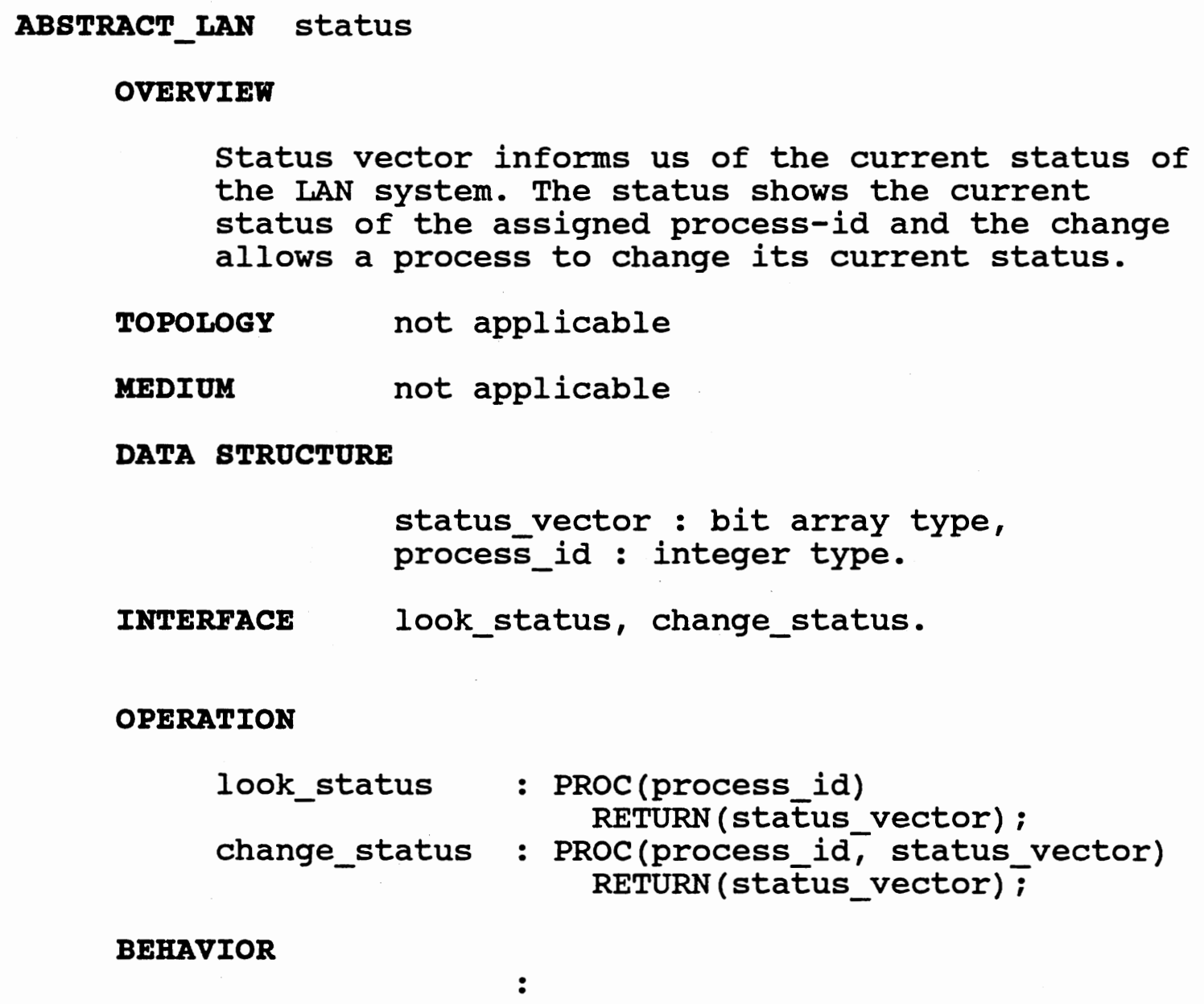

\section{END ABSTRACT_IAN}

Figure 17. Abstract Specification for IAN Status 
ABSTRACT_LAN file

\section{OVERVIEW}

In LAN, the files can be created or deleted by each entity and can be transmitted to each other and also copied among the entities in the IAN communication.

TOPOLOGY

not applicable

MEDIUM

not applicable

DATA STRUCTURE

file : logical records of the basic types, source : integer type, destination : integer type.

INTERFACE create_file, delete_file, copy_file, send_fille, recv_file.

OPERATION

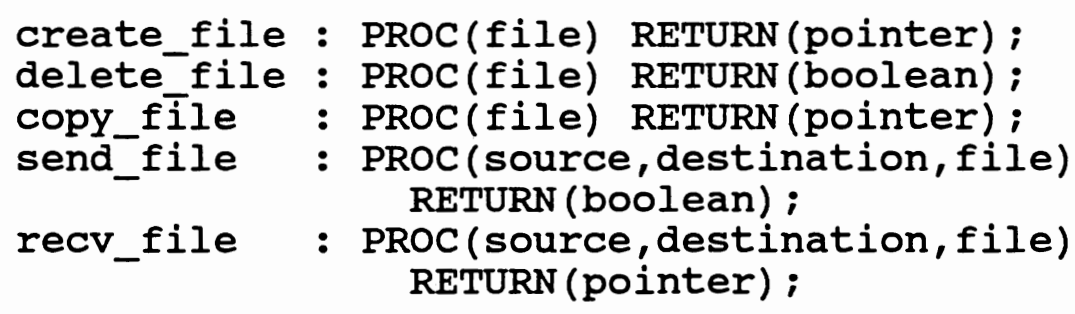

BEHAVIOR

\section{END ABSTRACT_IAN}

Figure 18. Abstract Specification for object File 
ABSTRACT_IAN message

\section{OVERVIEW}

Electronic mail can be created or deleted by each entity and can be transmitted to each other and also copied among the processes in the IAAN.

TOPOLOGY

not applicable

MEDIUM

not applicable

DATA STROCTURE

message : record type or file type, source : integer type, destination : integer type.

INTERFACE create_msg, delete_msg, copy_msg, send_msg, recv_msg.

OPERATION

create_msg : PROC(message) RETURN (pointer);

delete_msg : PROC(message) RETURN (boolean);

copy_msg : PROC(message) RETURN (pointer);

send_msg : PROC (source, destination, message) RETURN (boolean) ;

recv_msg : PROC(source, destination, message) RETURN (pointer);

BEHAVIOR

\section{END ABSTRACT_IAN}

Figure 19. Abstract Specification for Object Message 
ABSTRACT_IAN batch_job

\section{OVERVIEW}

In LAN, the batch processing job is possible among the processes in the IAN.

TOPOLOGY

not applicable

MEDIUM

not applicable

DATA STROCTURE

job : logical structure of record type or file type,

source : integer type,

destination : integer type,

host : integer type,

station : integer type.

INTERFACE send_job, recv_job, run_job.

OPERATION

send_job : PROC(source, destination, job) RETURN (boolean) ;

recv_job : PROC(source, destination, job)

run_job : PROC(host, station, job) RETURN (pointer) ;

RETURN (boolean) ;

BEHAVIOR

END ABSTRACT_IAN

Figure 20. Abstract Specification for object Batch_job 
ABSTRACT_LAN pdu(protocol data unit)

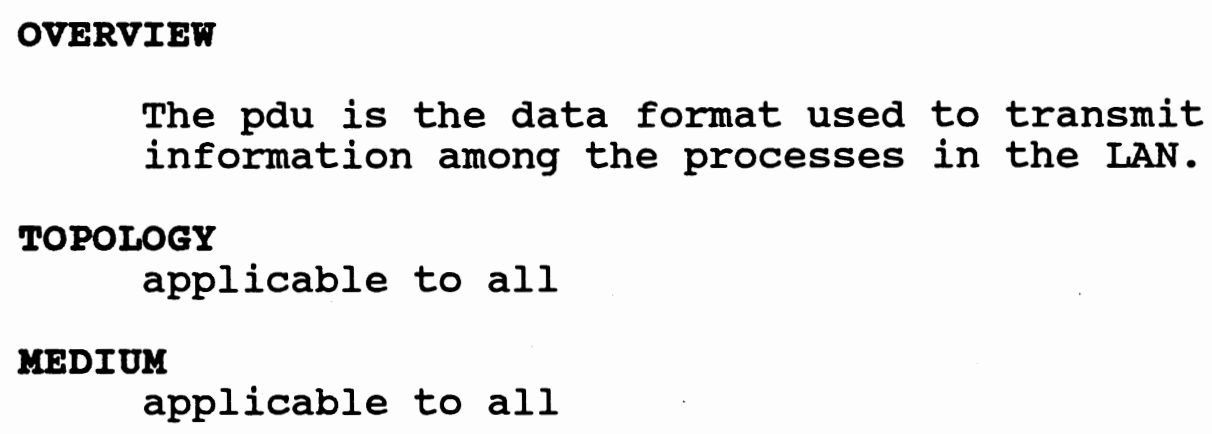

DATA STRUCTURE

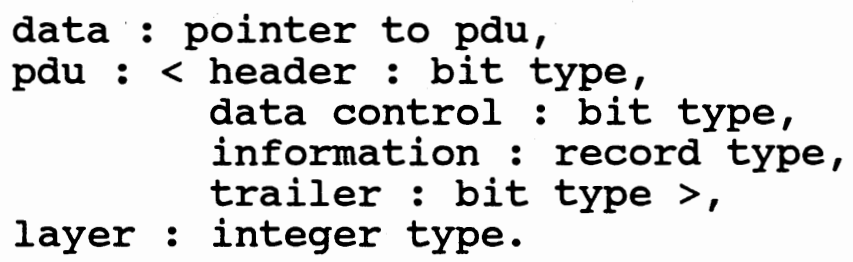

INTERFACE attach_header, detach_header, gen_header.

OPERATION

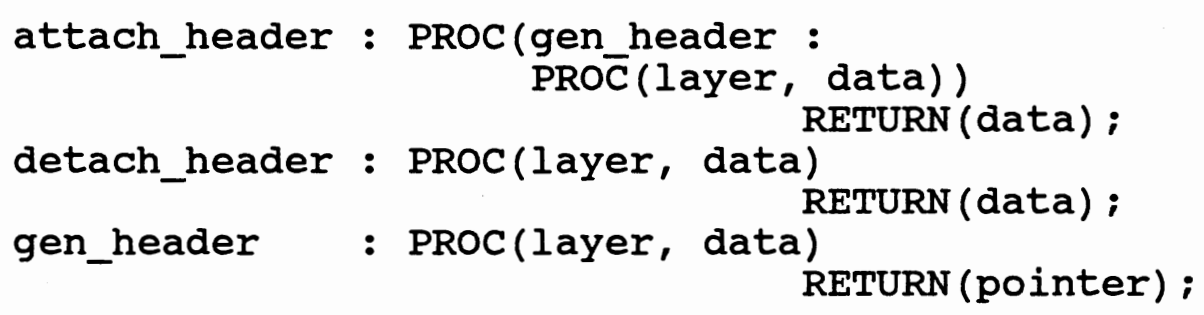

BEHAVIOR

END ABSTRACT_IAN

Figure 21. Abstract Specification for object PDU

Here we can see the procedure gen_header is used as the parameter of the procedure attach_header. 
ABSTRACT_LAN buffer

OVERVIEW

The buffer is for the buffering the information or the data inserted for synchronization when they are transmitted so that they are finally removed from a buffer for delivery.

TOPOLOGY

applicable to all

MEDIUM

applicable to all

DATA STRUCTURE

buffer : pointer type, data : pointer type.

INTERFACE insert, remove.

OPERATION

insert : PROC(data, buffer) RETURN (pointer); remove : PROC(data, buffer) RETURN(boolean);

BEHAVIOR

END ABSTRACT_IAN

Figure 22. Abstract Specification for object Buffer 
So far, we have specified some objects which can be implemented in a particular local area network. To do this, we left the abstraction for the protocols of each layer in LAN. The abstract specifications for the protocols in the existing IAN model are given in Chapter 6 and Appendixes. Now we confront the question of how we can use these abstraction modules. Figure 23 shows the relationship between the IAN abstract modules specified above and the service operations necessary in a certain LAN. In this figure, ' $\rightarrow$ A.B' means a function call to an abstract module for LAN object 'A' using its interface operation as a qualifier ' $B$ '. 


\begin{tabular}{|c|c|c|}
\hline service operation & & abstract operation \\
\hline system initiation & $-->$ & osu_lan.set_up \\
\hline request service & $-->$ & osu_lan.service \\
\hline status control & $-->$ & $\begin{array}{l}\text { status.look_status } \\
\text { status.change_status }\end{array}$ \\
\hline \multicolumn{3}{|l|}{ application } \\
\hline message transfer & $-->$ & $\begin{array}{l}\text { message. create_msg } \\
\text { message.send_msg } \\
\text { message.recv_msg }\end{array}$ \\
\hline file transfer & $-->$ & $\begin{array}{l}\text { file.create file } \\
\text { file.send_file } \\
\text { file.recv_file }\end{array}$ \\
\hline batch_job & $-->$ & $\begin{array}{l}\text { batch_job.send_job } \\
\text { batch_job.recv_job } \\
\text { batch_job.run_job }\end{array}$ \\
\hline synchronization & $-->$ & $\begin{array}{l}\text { buffer.insert } \\
\text { buffer.remove }\end{array}$ \\
\hline pdu manipulation & $-->$ & $\begin{array}{l}\text { pdu.attach_header } \\
\text { pdu.detach_header }\end{array}$ \\
\hline system termination & $\rightarrow$ & osu_lan.shut_down \\
\hline
\end{tabular}

Figure 23. Relations between Service and Abstraction

Combining all these LAN abstract modules, now we show how this LAN abstraction methodology can be applied to the existing LAN standard protocols. The details are provided in the next chapter. 


\section{CHAPTER VI}

\section{LAN ABSTRACT SPECIFICATION APPLICATION}

\subsection{Application to OSI Model LAN}

In a multivendor LAN environment, LAN standards are necessary to achieve compatibility. While the LAN related device companies have tried to collaborate for standards with some constraints for standardization such as economic interests, information security and political considerations, some users are also developing their own implementations using the LAN standards. It rids users of unnecessarily expensive and inefficient implementations. Among them, two big users are General Motors and Boeing company. General Motors developed a specification for communication standards in factory environment called Manufacturing Automation Protocol(MAP) [Kaminski 1986] and Boeing addressed a specification of communication network standards for the technical and office environment called Technical and office Protocols(TOP) [Farowich 1986]. The application of the TOP includes electronic mail, word processing, file transfer, database management and distributed batch jobs. Here, we show how the specification of the abstraction associated with the LAN standard 
protocol like MAP or TOP can be actually implemented in the IAN simulation program or LAN software using the abstract specification modules with some proper modifications. The IEEE 802.5 Token Ring and MAC protocol and its service function are used for the specification of the physical layer and the IEEE 802.2 Logical Link Control service and protocol are used for the specification of the data link layer. In this IAN specification, however, the network, transportation, session and presentation layers are supposed to be included in application layer. There is relatively tight relationship among these layers of the OSI model and those are not well defined for LAN. Each communication entity has its own protocol layers. With each of these, the abstractions for its service functions are specified in Appendix A. Also, we use some of the associated objects specified in the previous chapter which are necessary in a IAN system. The upper-level-layer abstraction in Appendix A shows the LAN application layer abstraction. Among the corresponding sub-layers in a certain local area network, the data link layer corresponds with the application layer as the highest sub-layer. Such IEEE 802.2 logical link control layer is shown in datalink-control abstraction in Appendix A. With LLC layer, we need a lowest layer protocol such as the IEEE 802.5 token ring. We need its peer MAC services and physical layer specifications. Abstractions of these are also specified in Appendix A. 
Using abstraction modules, we show an example of implementation. In connection-mode service, a service following the user application operation in a communication system consists of three phases [Stallings 1987]: (1) connection establishment, (2) data transfer, and (3) connection release. In Figure 24 , we show how the LAN abstract module works with the possible operations when the connection-mode service is offered in a LAN. Data transmission is one of the operations. The numbers on this figure are associated with those in Figure 25.

\begin{tabular}{|c|c|c|}
\hline service operation & \multicolumn{2}{|l|}{ abstract operation } \\
\hline system initiation & osu_lan.set_up( ) & \\
\hline service request & osu_lan.service(type) & \\
\hline in lan.service & & \\
\hline connection establishment & $\begin{array}{l}\text { application.a_associate.request() } \\
\text { application.a_associate.confirm() }\end{array}$ & $\begin{array}{l}-\cdots(1) \\
\leftrightarrow-(2)\end{array}$ \\
\hline $\begin{array}{l}+->\text { data transfer } \\
\mid \\
+--(\text { loop })\end{array}$ & $\begin{array}{l}\text { application.a_data.request () } \\
\text { application.a_data.confirm( ) }\end{array}$ & $\begin{array}{l}\cdots \text { (3) } \\
\langle-(4)\end{array}$ \\
\hline connection release & $\begin{array}{l}\text { application.a_release.request() } \\
\text { application.a_release.confirm() }\end{array}$ & 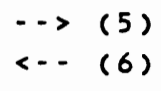 \\
\hline system termination & osu_lan.shut_down( ) & \\
\hline
\end{tabular}

Figure 24. Operations in Connection-Mode Service 
Figure 25 shows the peer entity operations with above operations. The numbers should be matched with peer operations (refer to Figure 24 also).

$\operatorname{user}(i) \quad \operatorname{user}(j)$

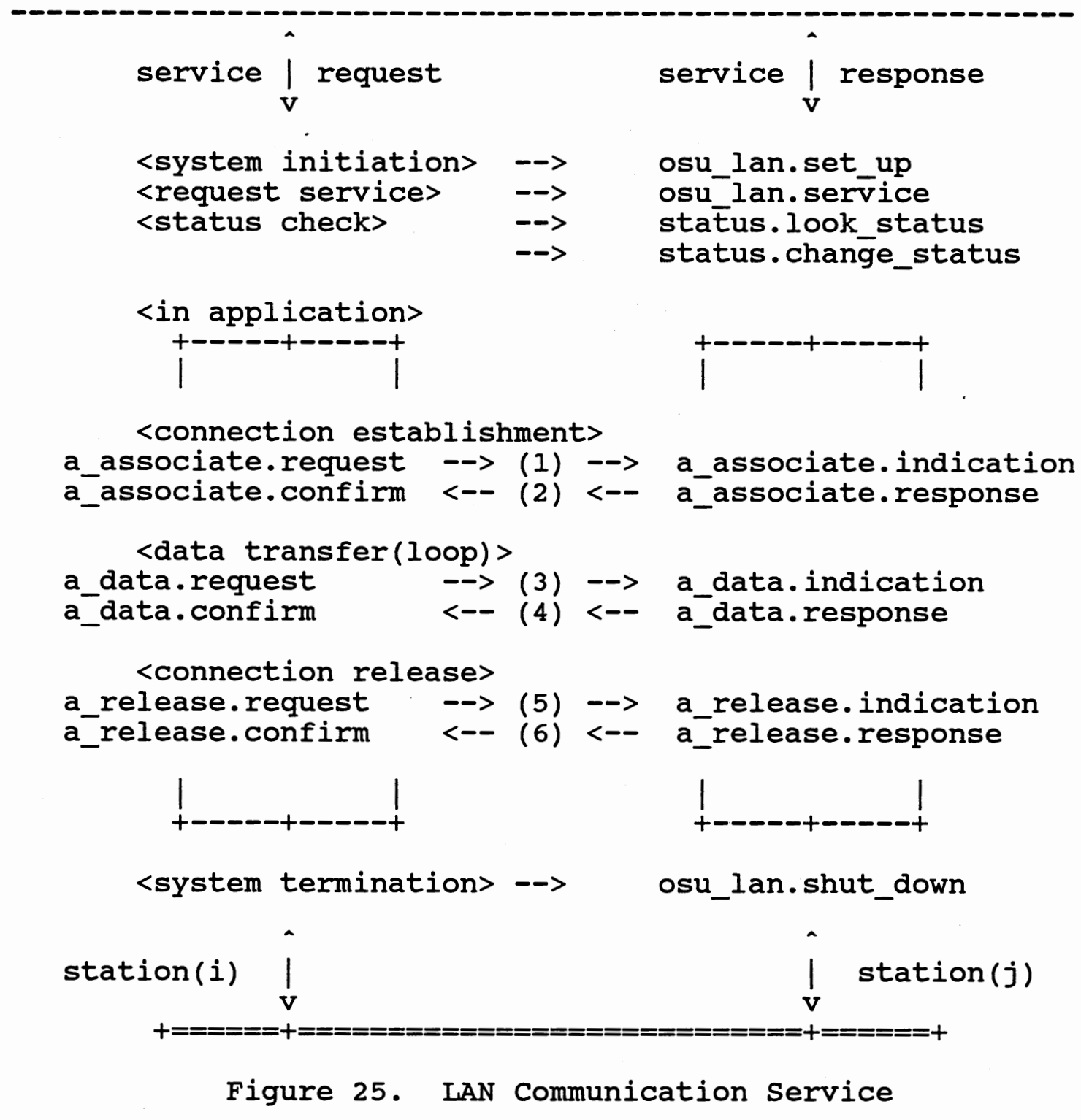


Now we examine the trace of service in detail when a LAN is invoked and requested to transfer data. Most of the codes consist of functional module call using abstracted module which specifies its function in abstract specification model and the associated codes. Figure 26 shows which service is necessary and how abstract module can be called. In this figure, the "->" sign means abstract module calling. 


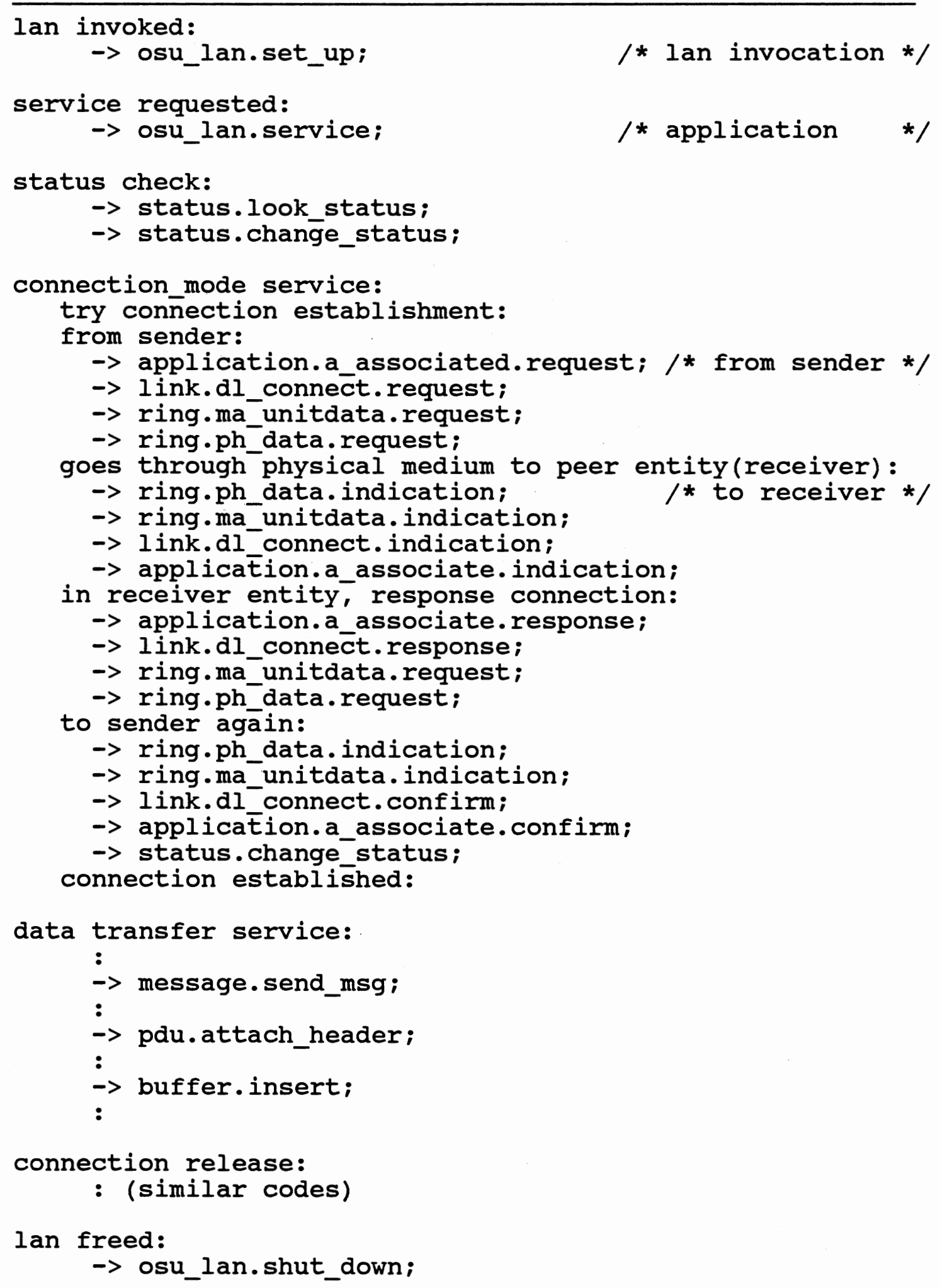

Figure 26. Trace of service 


\subsection{Application to DOD Model IAAN}

We have thought about the specification of OSI model LAN abstraction. We apply abstract specification model to another LAN structure, namely, the TCP/IP model. To do this, we show how this model can be applied to DOD computer network which uses TCP/IP as sub-layer protocols. The TCP/IP are mandatory for use in DOD packet switching networks [Defense 1983]. TCP provides similar services as the transport layer does in OSI model. It provides reliable connection-oriented communication between processes in networks. TCP requires IP as sub-layer protocol. The IP provides services to transport layer and relies on the services of the lower-layer protocols. For LAN, IEEE 802 standards can be a model of such lower-layer. Figure 27 shows DOD communication architecture for IAN. Abstract specification for DOD IAN Model is in Appendix B. 
$\underline{D O D}$

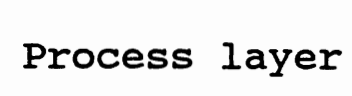

Host-to-Host

Internet

Network Access

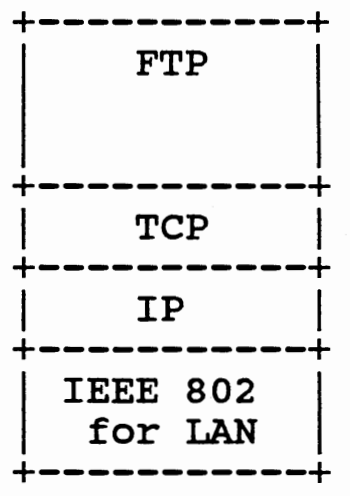

$\underline{O S I}$

application presentation session

transport

network

data link physical

Figure 27. DOD Communication Architecture for IAN 
How can DOD LAN abstraction modules be used? Figure 28 shows how abstract modules are invoked to offer the services in a DOD LAN model.

Service requested from port sender to port receiver: for connection establishment:

from sender:

$\rightarrow$ ulp.active_open(); /* ulp: Upper Level Protocols */

$\rightarrow$ tcp.active_open( $)$ /* tcp: Trans Control Protocol*/

$\rightarrow$ ip.send $/ *$ ip : Internet Protocol */

$\rightarrow$ llc.connect. request ();

/* llc: Lower Level Protocols */ control data goes through internet:

$\rightarrow$ ulp.wait(connection established);

from receiver:

$\rightarrow$ ulp.active_open(); or ulp.passive_open();

similar codes:

open matching:

connection established:

for data transfer:

from sender:

$\rightarrow$ ulp.send_data ();

$\rightarrow$ tcp.send ();

$\rightarrow$ ip.send ():

$\rightarrow$ lic.data.request ():

data goes through physical medium:

to receiver:

$\rightarrow$ llc.data.response ();

$\rightarrow$ ip.deliver();

$\rightarrow$ tcp.deliver ():

$\rightarrow$ ulp.deliver ():

data transferred:

acknowledgment:

connection termination:

similar codes:

Figure 28. Trace of DOD Communication 
CHAPTER VII

CONCLUSIONS

We have shown the abstraction methodology and its use in the specification of local area networks. We have presented a formal model for LAN communication in terms of the inter-layer transmission and an abstract IAN specification template. Using this abstract specification template, all levels of communication protocols are defined. Each of these is an abstract module which contains each service function of each protocol layer. An abstract module also can define an object used in a LAN system. To use each object, its abstract module is called. Data, procedural and behavioral abstractions of the LAN objects are specified with this object module. How the abstract specification can be used when it is implemented is illustrated by tracing the service function calling and showing the inter-relations between the abstract modules. The OSI Basic Reference Model and IEEE 802 IAN standard protocols have been chosen as examples for LAN abstract specifications. The same abstraction model has been applied to TCP/IP in DOD packet switching network model for showing the usability and compatibility of the abstraction model. LAN simulation using this abstract specification is 
one of the possible topics for research. To do this implementation, the selection of the proper programming language and the other decisions such as the process state transition, data packet format, error condition and the buffer size considering the LAN performance are prerequisite. The selection of a high-level language for the implementation of a LAN abstract specification module is also a topic for research. Other research topics include network operating systems, network software and network user interface commands with their interpreter and compiler using their abstract specification schemes. 


\section{BIBLIOGRAPHY}

Berntsen, J.A., Davin, J.R., Pitt,D. A., and Sullivan, N.G. MAC layer interconnection of IEEE 802 local area networks. Computer Networks and ISDN Systems 10, $5(1985), 259-273$.

Berztiss, A. T. and Thatte, S. Specification and implementation of abstract data types. Advances in Computers, 22 (1983) Academic Press, 295-353.

Blumer, T. P. and Tenney, R. I. A formal specification technique and implementation method for protocols. Computer Networks 6, 3(1982), 201-217.

Bochmann, G. V., Finite state description of communication protocols. Computer Networks 2, 4/5(1978), 361-372.

Bochmann, G. V. and Sunshine, C. A. Formal methods in communication protocol design. IEEE Trans. on Comm. COM-28, 4(1980), 624-631.

Cleaveland, J. C. An Introduction to Data Types. Addison-Wesley Publishing Co.(1986).

Comer, Douglas. Operating System Design - Vol. 2 . Internetworking with Xinu. Prentice-Hall, Inc.(1987).

Dasgupta, S. Computer design and description languages. Advances in Computers,21(1982), Academic Press, 91-154.

Derfler, F. J. Jr. and Stallings, W. A Manager's Guide to Local Networks. Prentice-Hall, Inc., NJ(1983).

Defense Communication Agency. Military standard: Internet Protocol. MIL-STD-1777(1983)., Transmission Control Protocol. MIL-STD-1778(1983) ., File Transfer Protocol. MIL-STD-1780(1984) ., Simple Mail Transfer Protocol. MII-STD-1781(1984) ., Telnet Protocol. MIL-STD-1782 (1984).

Ehrich, H. D. On the theory of specification, implementation, and parametrization of abstract data types. JACM, Vol.29, No.1(January 1982), 206-227. 
Engels, C., Pletat, U., and Ehrich H.-D. An operational semantics for specifications of abstract data types with error handling. Acta Informatica 19, 3(1983), 235-253.

Farowich, S. A. Communicating in the technical office. IEEE Spectrum Vol.23 (April 1986), 63-67.

Gehani, N. and McGettrick, A. D. Software Specification Techniques. Addison-Wesley, International Computer Science Series(1986)

Graube, M. and Mulder, M. C. Local area networks. IEEE Computer, Vol.17, No.10 (Oct. 1984), 242-247.

Guttag, J. V. and Horning J. J. The algebraic specification of abstract data types. Acta Informatica 10, 1(1978), 27-52.

Guttag, J. V., Horowitz, E. and Musser, D. R. Abstract data types and software validation. Comm. of the ACM 21, 12(1978), 1048-1064.

Guttag, John V. Notes on type abstraction(Version 2). IEEE Trans. on Software Eng., Vol.SE-6, 1 (Jan. 1980), 13-32.

Hawe, B., Kirby, A. and Lauck, A. An architecture for transparently interconnecting IEEE 802 LAN. DEC-TR-322, Digital Equipment Corporation(1984) .

Hawe, W. R. and Varghese, G. Extended local area network management principles. DEC-TR-324, Digital Equipment Corporation(1984) •

ISO/TC97/SC16. Data Processing - Open Systems Interconnection - Basic Reference Model. ISO/TC97/SC16, ANSI, 1430 Broadway, New York, NY. Computer Networks 5,1(1981), 81-118.

Kaminski, M. A. Jr. Protocols for communicating in the factory. IEEE Spectrum Vol.23 (April 1986), 56-62.

Keller, R. M. Formal verification of parallel programs. Comm. of the ACM 19, 7(1976), 371-384.

Kessler, G. Ethernet vs. IEEE 802.3. LAN Magazine (July 1987).

Kummer, P., Tasker, R., Linge, N. and Ball, E. A protocolless scheme for bridging between IEEE 802 Local Area Networks. Computer Networks and ISDN Systems 12 , $2(1987), 81-87$. 
Lam, Simon S. Tutorial: Principles of Communication and Networking Protocols. IEEE Computer Society, IEEE Catalog No. EHO216-2 (1984).

Liskov, B. and Guttag J. Abstraction and Specification in Program Development. The MIT Press, McGraw-Hill Book Co.(1986).

Liskov, B. and zilles, S. Specification techniques for data abstractions. IEEE Trans. on Software Eng. $1,1(1975)$, 9-19.

Mclean, J. A formal method for the abstract specification of software. JACM, Vol.31, No.3 (July 1984), 600-627.

McQuillan, J. M. Local network technology and the lessons of history. Computer Networks 4,5(1980), 235-238.

Merlin, P. M. Specification and validation of protocols. IEEE Trans. on Comm. COM-27, 11(1979), 1671-1680.

Milner, Robin. A Calculus of Communicating systems. Springer-Verlag, Berlin, Lecture Notes in Computer Science $92(1980)$.

Nourani, C. F.

Abstract implementations and their correctness proofs. JACM, Vol.30,No.2 (April 1983), 343-359.

Saltzer, J. H., Pogran, K. T. and Clark, D. D. Why a ring? Computer Networks 7,4(1983), 223-231.

Sincovec, R. F. and Wiener, R. S. Data structures using Modula-2. John Wiley \& Sons, New York(1986).

Stallings, William. Data and Computer Communications. Macmillan Publishing Co. New York(1985).

Stallings, William. Handbook of Computer-Communications Standards. Vol.1 The open Systems Interconnection(OSI) Model and OSI-Related Standards, Vol.2 Local Networks Standards and Vol.3 Department of Defense(DOD) Protocol standards. Macmillan Publishing Co. New York (Vol.1,2 : 1987, Vol.3 : 1988).

stallings, William. Local networks. Computing Survey, Vol.16, No.1(March 1984),3-41.

Stallings, william. Local Networks. 2nd Ed. Macmillan Publishing Co. New York(1987).

Stallings, William. Tutorial: Local Network Technology. 2nd Ed. IEEE Computer Society, IEEE Catalog No. EHO234-5 (1985). 
Sunshine, C. A. and Dalal, Y. K. Connection management in Transport protocols. Computer Networks 2, 6(1978), 454-473.

Sunshine, C. A. Formal Modeling of Communication Protocols. ISI/RR-81-89, USC (March 1981).

Sunshine, C. A. Formal techniques for protocol specification and verification. Tutorial: Principles of communication and networking protocols. IEEE

Computer Society (ISBN 0-8186-0582-0), (1984), 467-473.

Tanenbaum, A. S. Computer Networks. Prentice-Hall, New Jersey(1981).

Tanenbaum, A. S. Network protocols. ACM Computing Surveys, Vol.13,No.4 (December 1981), 453-489.

Wirsing, M., Pepper, P., Partsch, H., Dosch, W., and Broy, M. On hierarchies of abstract data types. Acta Informatica 20,1(1983), 1-33. 
APPENDIXES 


\section{APPENDIX A}

OSI MODEL ABSTRACT MODULES 
For upper level layer(ISO Application Layer):

ABSTRACT_IAN application

OVERVIEW

Application layer is the highest layer in a network system. Application layer offers the service of user oriented high level protocol such as electronic mail, a file transfer, and remote job manipulation.

TOPOLOGY ring

MEDIUM twisted pair

DATA STRUCTURE

pdu : pointer type, calling p_id : integer type, called $p$ id : integer type, application_context : pointer type, responding $\bar{p}$ id : integer type, result: bit type, user_information : bit type.

INTERFACE

a_associate.request, a_associate.indication,

a_associate.response, a_associate.confirm,

a_data.request, a_data.indication,

a_data.response, a_data.confirm,

a_release.request, a_release.indication,

a_release.response, a_release.confirm,

OPERATION

PROC a_associate.request(calling p_id : INTEGER; called p_id : INTEGER; application context : POINTER);

PROC a_associate.indication(calling p_id : INTEGER; called p_id : INTEGER; application_context: POINTER);

PROC a_associate.rēsponse(responding p_id : INTEGER; application_context: PŌINTER; result : $\mathrm{BIT}$ );

PROC a_associate.confirm(responding p_id : INTEGER; application_context: POINTER; result : $\mathrm{BIT}$ );

PROC a_data.request (calling p_id : INTEGER; called p_id : INTEGER; pdu : POINTER);

PROC a_data.indication(calling p_id : INTEGER; called p_id : INTEGER; pdu : POINTER);

PROC a_data.response(calling p_id : INTEGER; called p_id : INTEGER; result: BIT);

PROC a_data.confirm(calling p_id : INTEGER; called p_id : INTEGER; result : BIT);

PROC a_release.request (user_information : BIT);

PROC a_release.indication(user_information:BIT) ; 
PROC a_release.response(user_information : BIT; result : $\mathrm{BIT})$;

PROC a_release.confirm(user_information : BIT; result : $\mathrm{BIT})$;

\section{BEHAVIOR}

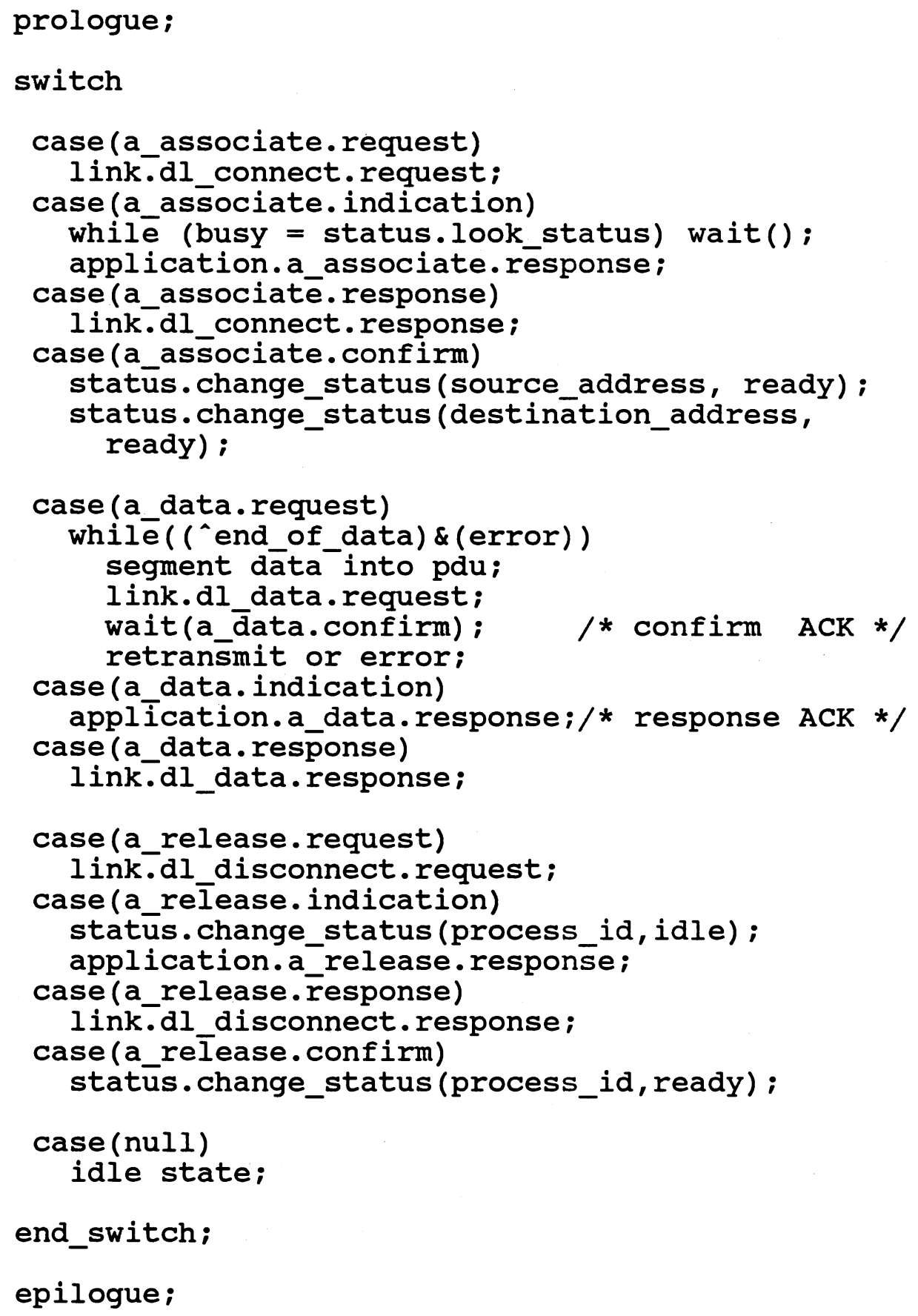


For data link control (IEEE 802.2 Logical Link Control):

\author{
ABSTRACT_IAN link /* IEEE 802.2 */ \\ OVERVIEW \\ Logical link control(LLC) is the highest layer in \\ a single local area network architecture. LLC \\ layer provides connection oriented service \\ between LLC users across a MAC controlled link. \\ TOPOLOGY ring \\ MEDIUM twisted pair \\ DATA STRUCTURE \\ pdu : pointer type, source_address: integer type, \\ destination_address : integer type, \\ priority : integer type, data : pointer type, \\ amount : integer type, status : bit type.
}

\title{
INTERFACE
}

dl_connect.request, dl_connect.indication,

dl_connect.response, dl_connect.confirm,

dl_data.request, dl_data.indication,

dl_data.response, dl_data.confirm,

dl_disconnect.request, dl_disconnect.indication,

OPERATION

PROC dl_connect.request(source_address :

INTEGER; destination_ad̄̄ress : INTEGER; priority : INTEGER);

PROC dl_connect.indication(source_address : INTEGER; destination_addresss : INTEGER; priority : INTEGER);

PROC dl_connect.response(source_address : INTEGER; destination_address : INTEGER; priority : INTEGER);

PROC dl_connect.confirm(source address : INTEGER; destination_ad̄̄ress : INTEGER; priority : INTEGER);

PROC dl_data.request(source_address : INTEGER; destination_address : INTEGER ; data : POINTER);

PROC dl_data.indication(source address : INTEGER; destination_ad̄̄ress : INTEGER; data : POINTER);

PROC dl_data.response(source_address : INTEGER; destination_address: INTTEGER; status:BIT);

PROC dl_data.confirm(source_address : INTEGER; destination_address: INTEGER; status:BIT) ; 


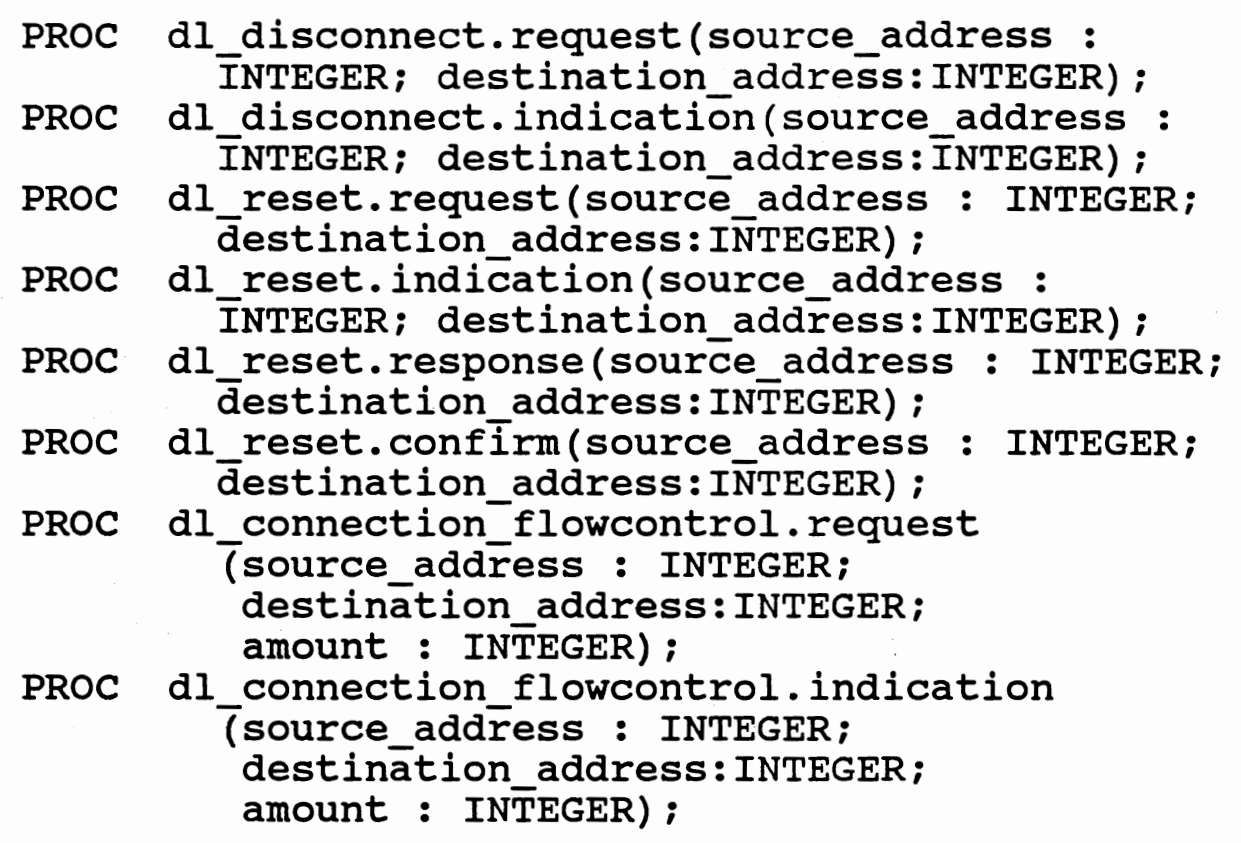

BEHAVIOR

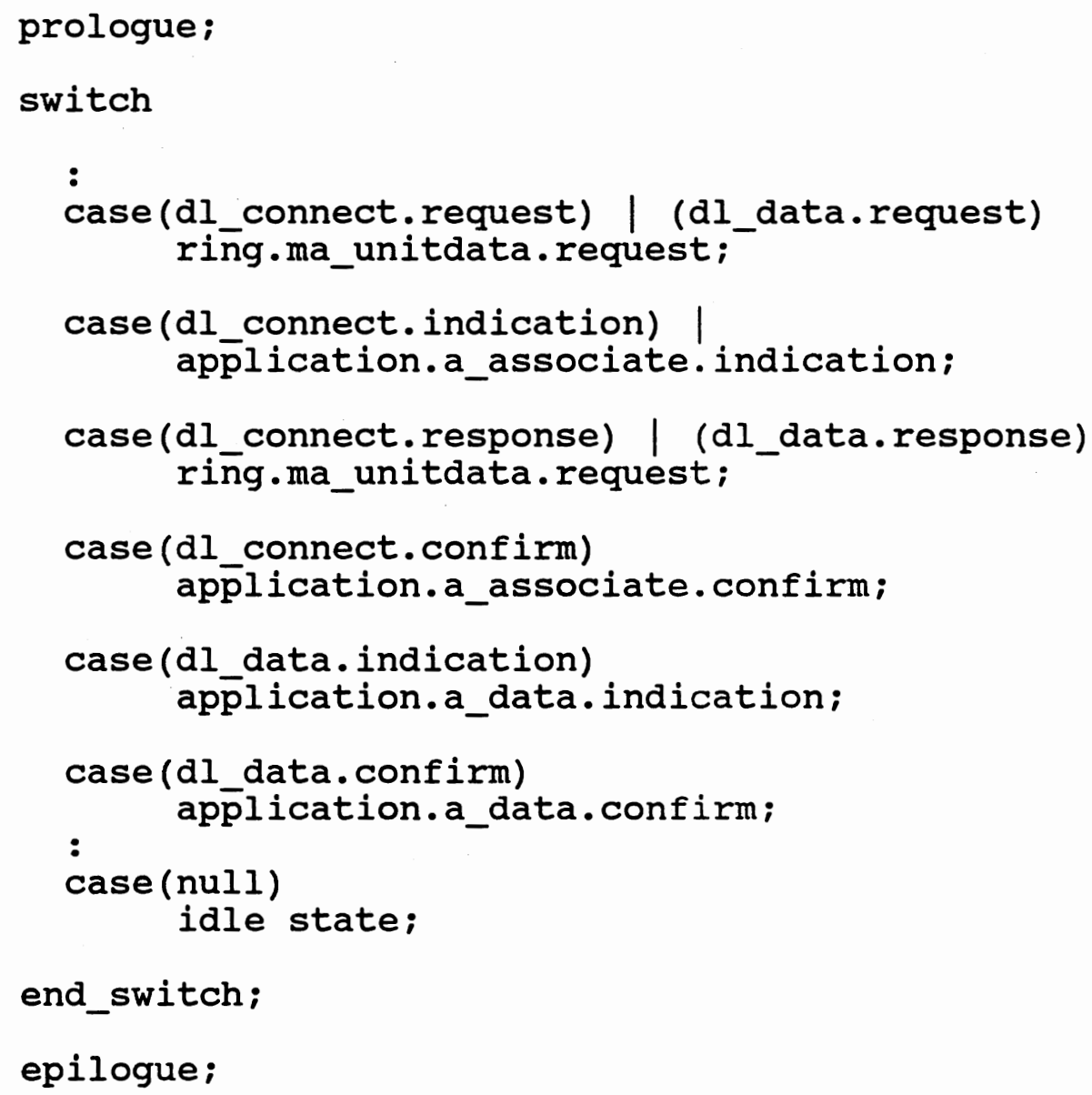


For medium access control (IEEE $802.5 \mathrm{MAC}$ ) and physical layer (IEEE 802.5 Token Ring):

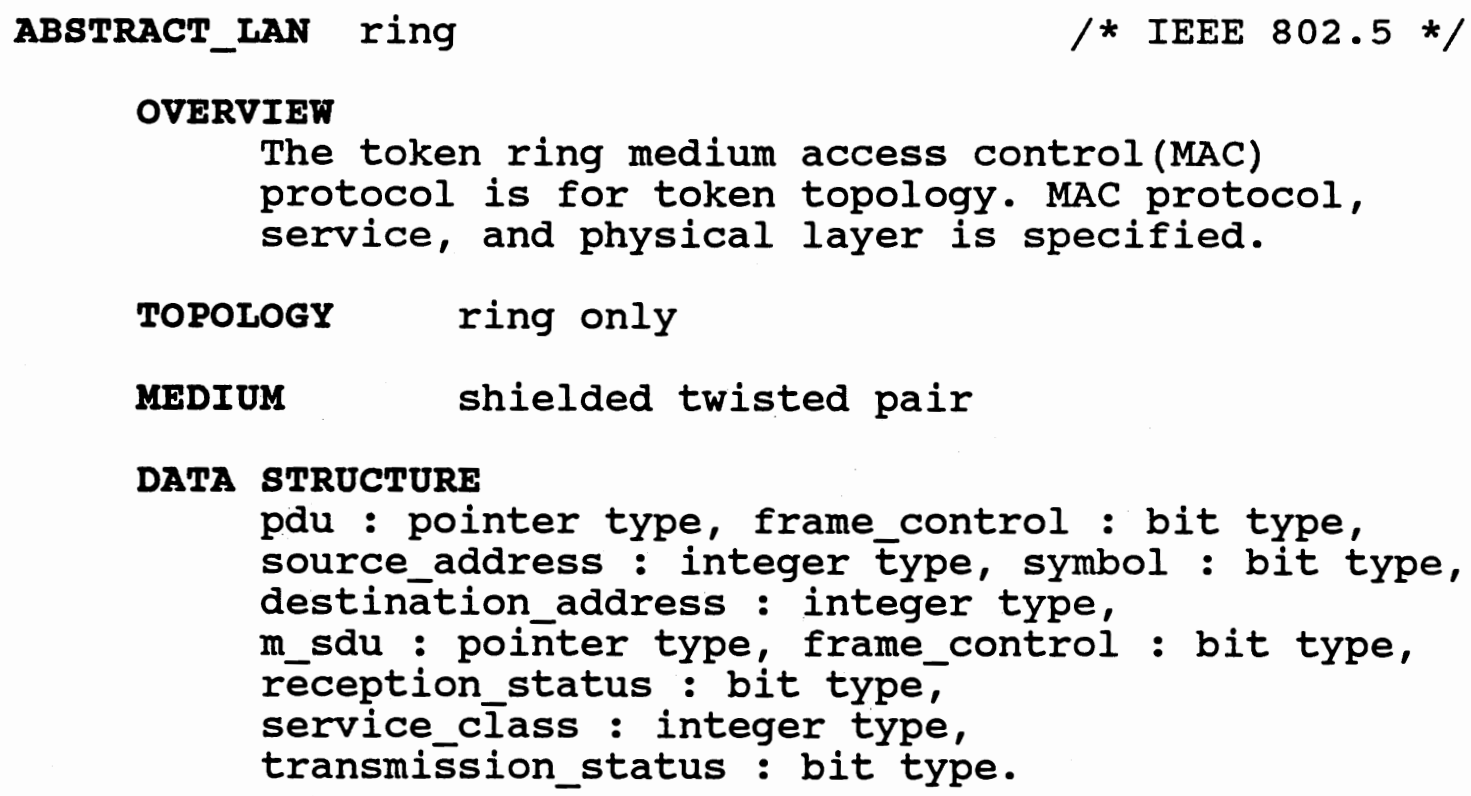

DATA STRUCTURE

pdu : pointer type, frame_control : bit type, source_address : integer Eype, symbol : bit type, destination_address : integer type, m_sdu : pointer type, frame_control : bit type, reception status : bit type, service_class : integer type, transmission_status : bit type.

\section{INTERFACE}

ma_unitdata.request, ma_unitdata.indication, ma_unitdata.status,

ph_data.request, ph_data.indication, ph_data.confirmation.

\section{OPERATION}

PROC ma_unitdata.request(frame_control: BIT; destination_address: INT̄EGER; m_sdu : POINTTER; sêrvice_class : INTEGER);

PROC ma unitdata.indication (frame_control: BIT; destination_address: INTEGER; source address : INTEGER m_sdu : POINTER; reception_status: BIT);

PROC ma_unitdata.status (transmission_status: $\bar{B} I T$; service_class : INTEGER);

PROC ph_data.request (symbol: BIT);

PROC ph_data.indication(symbol: BIT); PROC ph_data.confirmation(symbol: BIT); 
BEHAVIOR

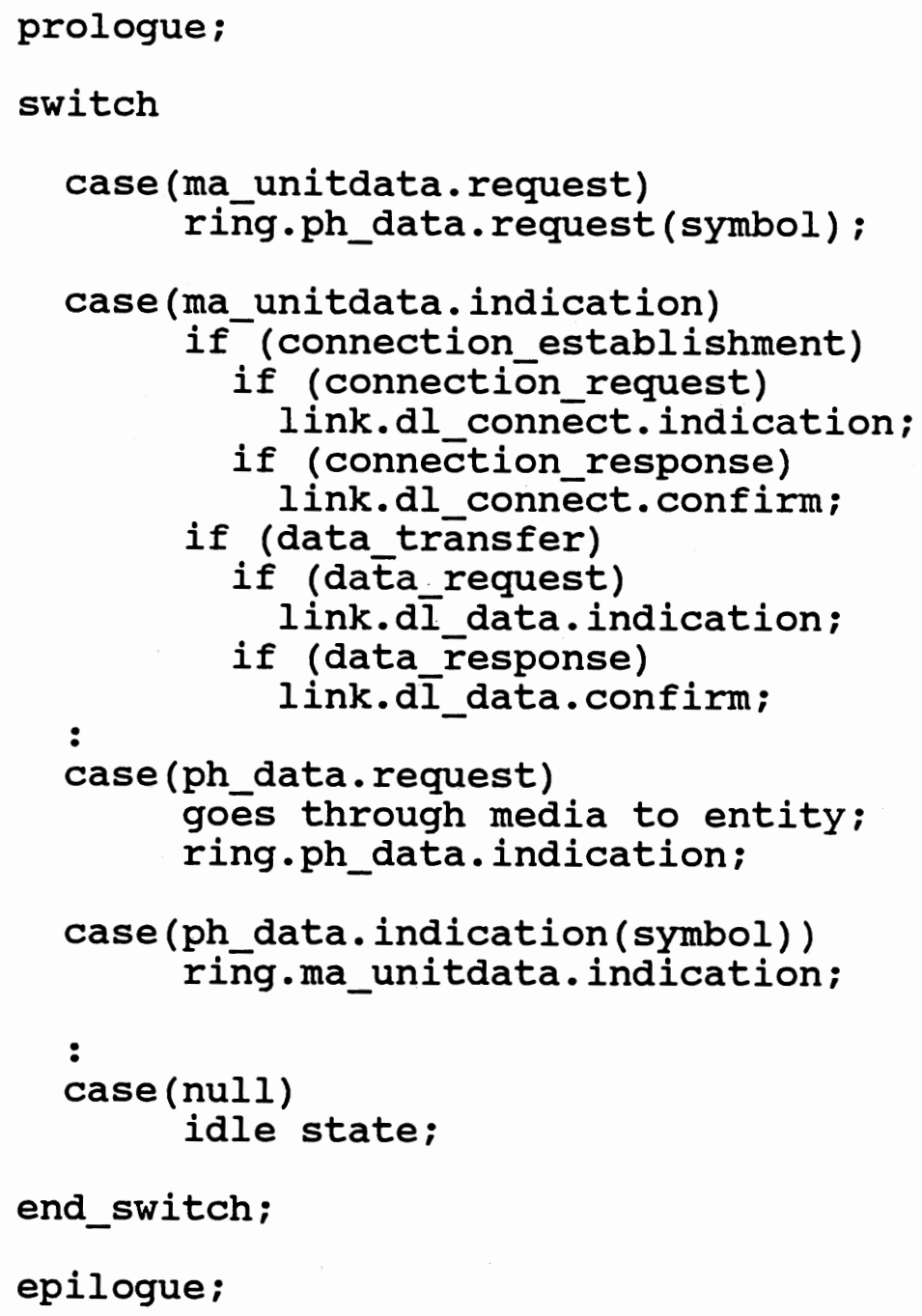


APPENDIX B

DOD MODEL ABSTRACT MODULES 
For upper level layer (process layer):

ABSTRACT_LAN ulp(upper level protocol)

OVERVIET

ULP is high level protocol like file transfer protocols. FTP(File Transfer Protocol) supports file transfer between processes in communication networks. It provides high level service associated with the next lower transport layer protocols.

TOPOLOGY ring, bus, star

MEDIUM twisted pair, coaxial cable, fiber optic cable

DATA STRUCTURE

source_address : integer type, destinātion_address : integer type, file : pointer to record type source_port : integer type, destination_port : integer type, local_connection_name : integer type, data : pointer to record type, data_length : integer type, connection_state : integer type, description : integer type, passive/active_flag : boolean type, close/abort_flāg : boolean type.

INTERFACE

connection, send_data, termination, check_status, open_ID, open_faìlure, open_success, deliver_data, closing, terminate, status_response, error.

\section{OPERATION}

PROC connection(source_port : INTEGER; destination port : INTEGER; passive/active_flag : BIT);

PROC send_data(local_connection_name : INTEGER; data : POINTER) ;

PROC termination(local_connection_name: INTEGER;

close/abort_flag : BIT) ;

PROC check_status(local_connection_name : INTEGER) ; 


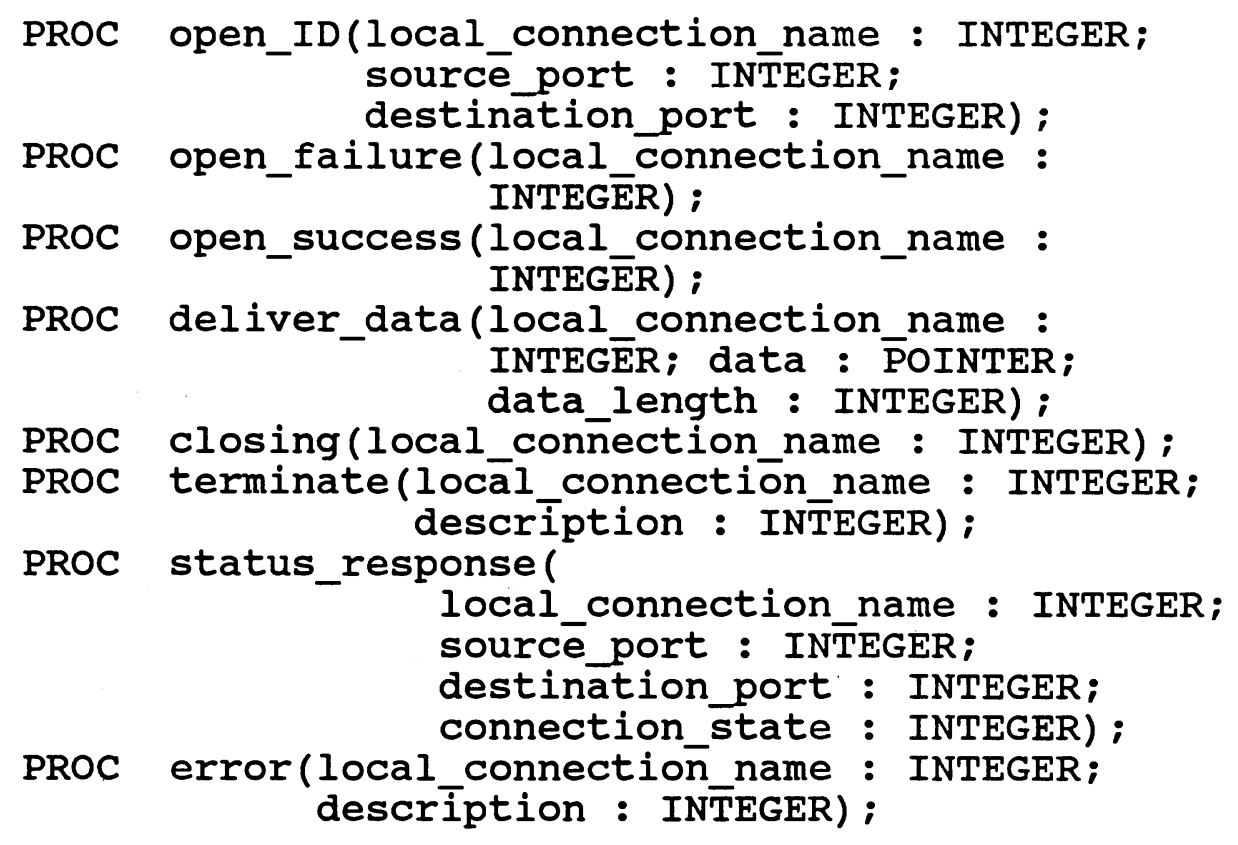

\section{BEHAVIOR}

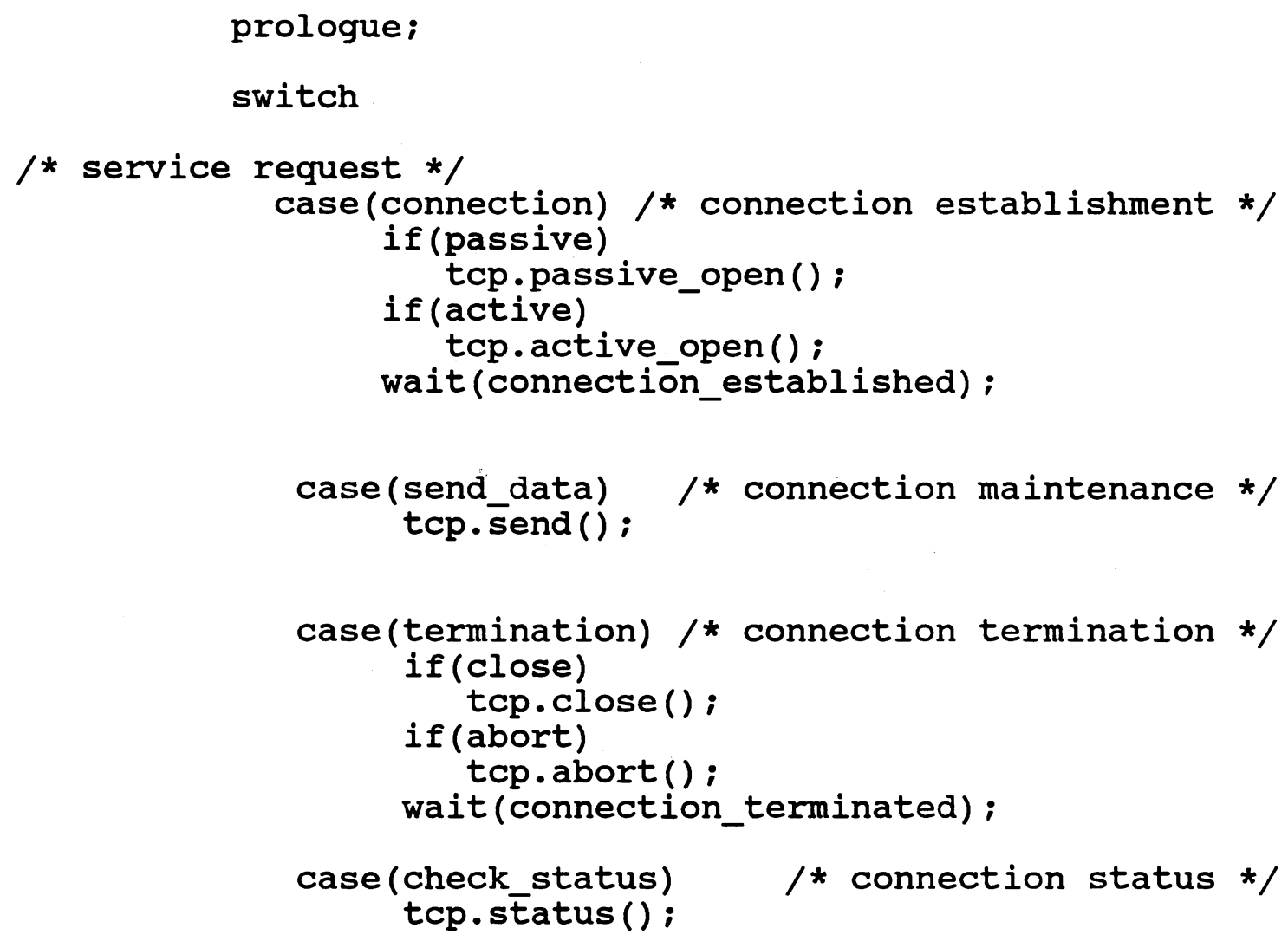




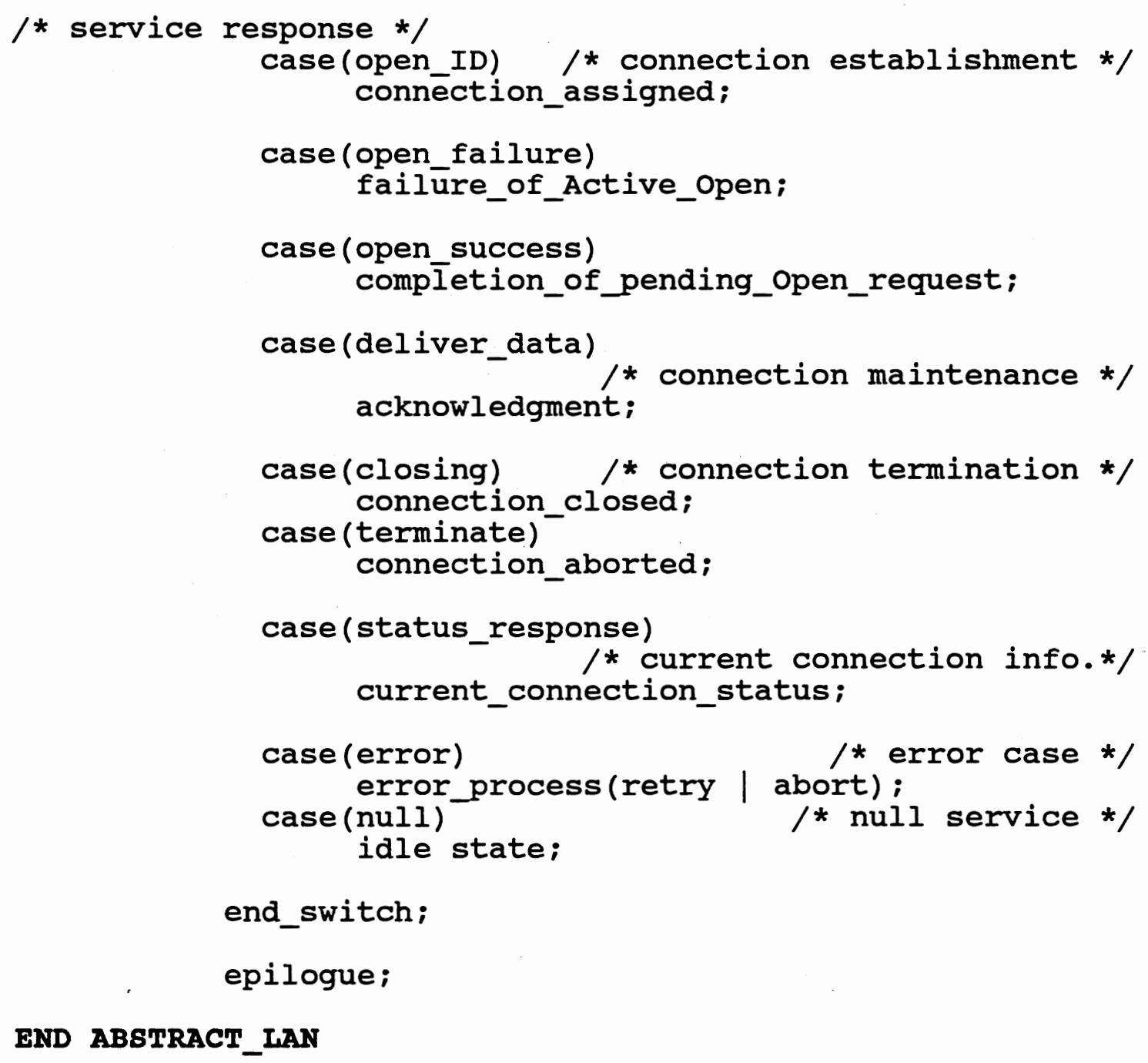


For TCP(Transmission Control Protocol):

\author{
ABSTRACT_IAN tcp \\ OVERVIEW \\ TCP is connection_oriented transport protocol \\ for use in packet switched communication networks \\ in which data trañsfer is reliable, ordered, \\ full_duplex, and flow controlled. \\ TOPOLOGY ring, bus, star \\ MEDIUM \\ twisted pair, coaxial cable, fiber optic \\ cable
}

DATA STRUCTURE

source port : integer type, destination port : integer type, local_connection name : integer type, data : pointer to record type, data_length : integer type, connection_state : integer type, description : integer type.

\title{
INTERFACE
}

/* service request primitives */ passive open, active open, send, allocate, closēe, abort, status /* service response primitives*/ open_ID, open_failure, open_success, deliverer, closing, terminate, status_response, error.

\section{OPERATION}

PROC passive_open(source_port : INTEGER;
destination_port : INTEGER);
PROC active_open(source_port : INTEGER;
destination_port : INTEGER);
PROC send(local_connection_name : INTEGER;
data : POINTER);
PROC close(local_connection_name : INTEGER);
PROC abort(local_connection_name : INTEGER);
PROC status(local_connection_name : INTEGER);
PROC open_ID(local_connection_name : INTEGER;
source_port : INTEGER;
destination_port : INTEGER);
PROC open_failure(local_connection_name :
INTEGER);




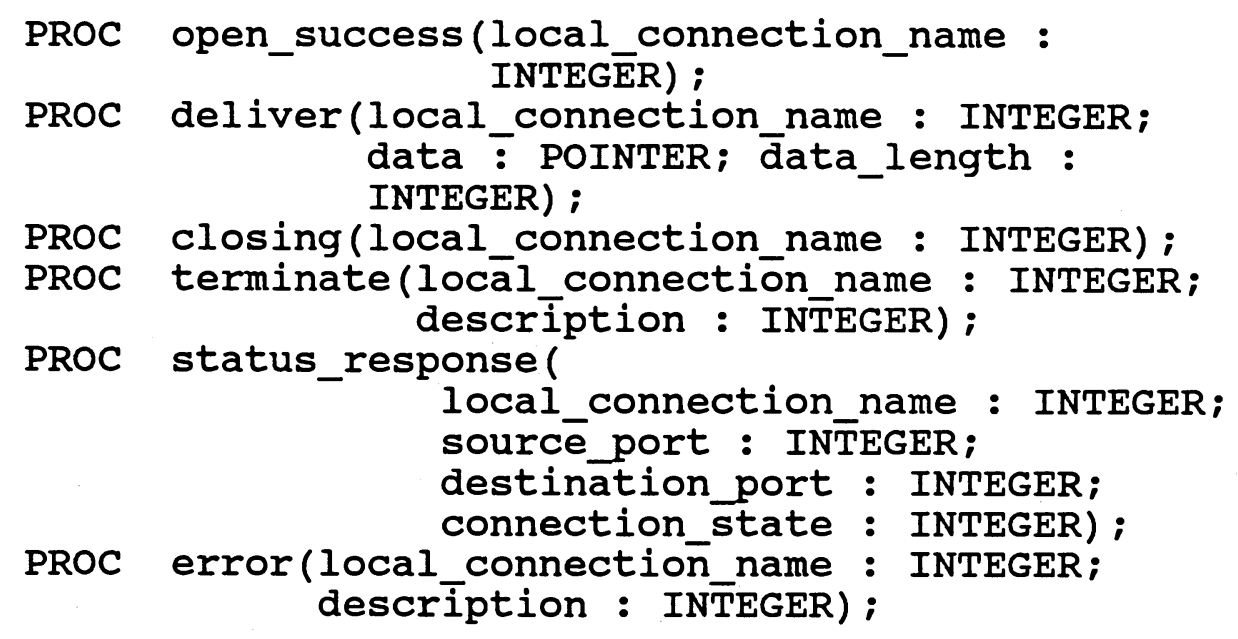

\section{BEHAVIOR}

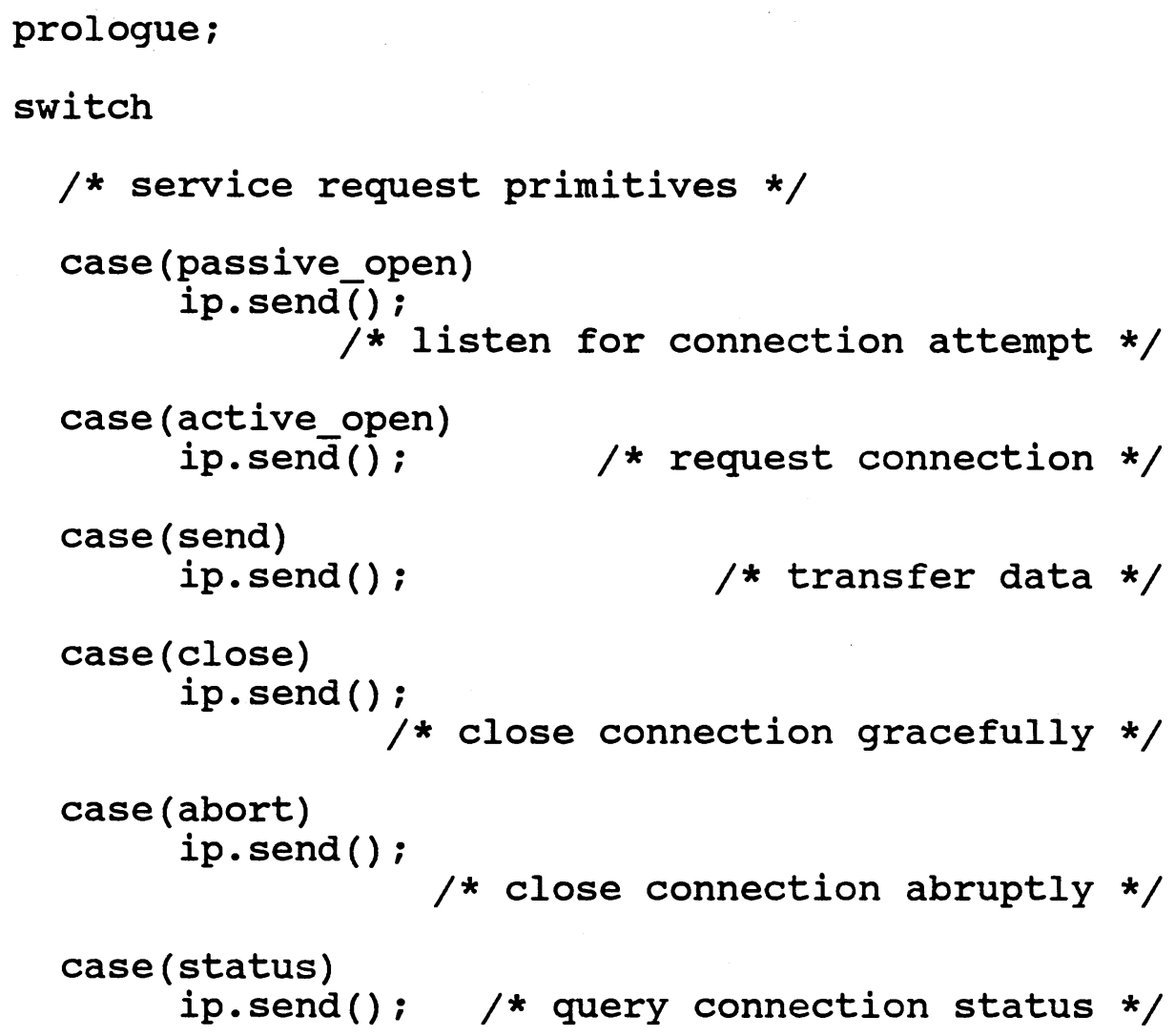




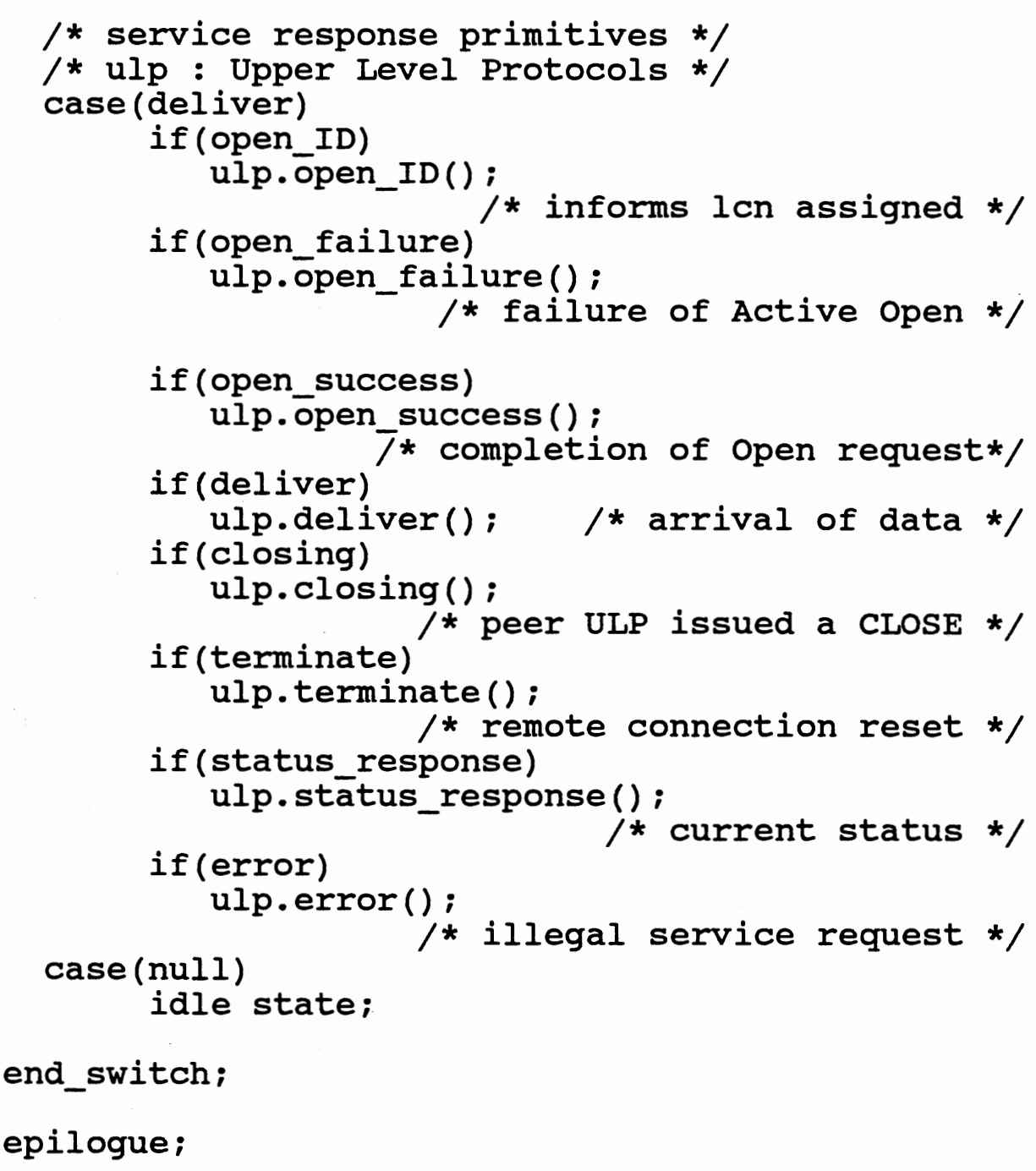

\section{END ABSTRACT_IAN}


For IP(Internet Protocol):

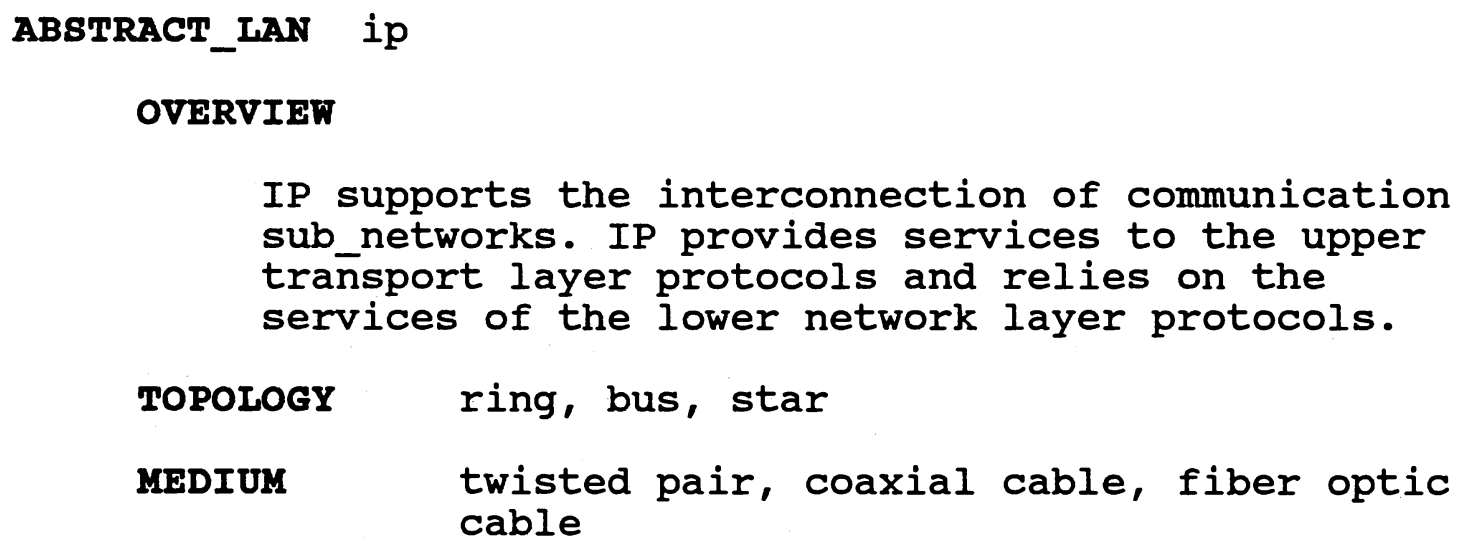

\title{
DATA STRUCTURE
}

source_port : integer type, destination_port : integer type, local_connection_name : integer type, data : pointer to record type, data_length : integer type, connection_state : integer type, description : integer type.

\section{INTERFACE}

send, deliver.

\section{OPERATION}

\author{
PROC send(source_address : INTEGER; \\ destinātion_address : INTEGER; \\ data_length ${ }^{-}$: INTEGER; \\ data : POINTER); \\ PROC deliver(source_address : INTEGER; \\ destination_address : INTEGER; \\ data_length : INTEGER; \\ data : POINTER);
}




\section{BEHAVIOR}

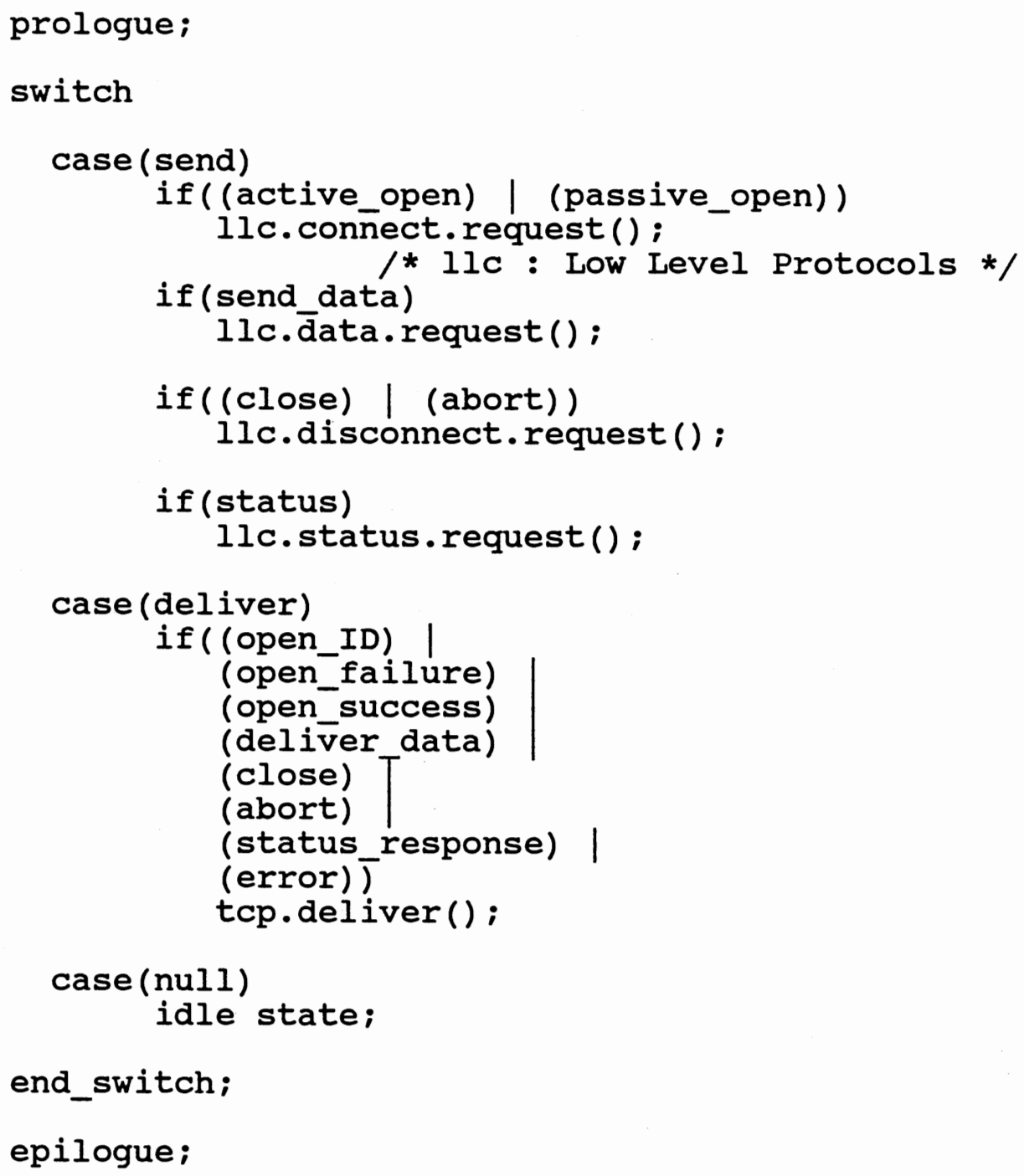


For sub layers:

Similar codes with IEEE 802 LAN and the OSI Reference Model in Appendix A. 
VITA

Chang-Hyun Jo

Candidate for the Degree of

Master of Science

Thesis: ABSTRACTION AND SPECIFICATION OF LOCAL AREA NETWORKS

Major Field: Computing and Information Sciences

Biographical:

Personal Data: Born in Pusan, Korea, April 25, 1958, the son of Soon-Kyu and Boon-Sun Jo.

Education: Graduated from Myong Ji Senior High

School, Seoul, Korea, in February, 1976; received Bachelor of Economics in statistics from sung Kyun Kwan University, Seoul, Korea, in February, 1984; completed requirements for the Master of Science degree at Oklahoma state University in July, 1988 .

Professional Experience: Software Engineer, Electronic Research Lab., Hyo Sung Corp., Seoul, Korea, December, 1983, to April, 1985; Teaching Assistant, Department of Computing and Information Sciences, Oklahoma State University, August, 1987, to July, 1988; 